\title{
Heparanase from triple-negative breast cancer and platelets acts as an enhancer of metastasis
}

\author{
WEN-JING YANG ${ }^{1}$, GAN-LIN ZHANG $^{1}$, KE-XIN CAO $^{1}$, XIAO-NI LIU ${ }^{2}$, \\ XIAO-MIN WANG ${ }^{1}$, MING-WEI YU ${ }^{1}$, JIN-PING LI $^{3}$ and GUO-WANG YANG ${ }^{1}$ \\ ${ }^{1}$ Department of Oncology, Beijing Hospital of Traditional Chinese Medicine, Capital Medical University, \\ Beijing 100010; ${ }^{2}$ Beijing Institute of Hepatology, Beijing Youan Hospital, Capital Medical University, \\ Beijing 100069, P.R. China; ${ }^{3}$ Biomedical Center, Uppsala University, Uppsala 75123, Sweden
}

Received March 11, 2020; Accepted July 2, 2020

DOI: 10.3892/ijo.2020.5115

\begin{abstract}
Triple-negative breast cancer(TNBC), which is characterized by inherently aggressive behavior and lack of recognized molecular targets for therapy, poses a serious threat to women's health worldwide. However, targeted treatments have yet to be made available. A crosstalk between tumor cells and platelets (PLT) contributing to growth, angiogenesis and metastasis has been reported in numerous cancers. Heparanase (Hpa), the only mammalian endoglycosidase that cleaves heparan sulfate, has been demonstrated to contribute to the growth, angiogenesis and metastasis of numerous cancers. Hypoxia affects the growth, angiogenesis and metastasis of nearly all solid tumors, and the ability of Hpa to promote invasion is enhanced in hypoxia. However, whether Hpa can strengthen the crosstalk between tumor cells and PLT, and whether enhancing the biological function of Hpa in TNBC promotes malignant progression, have yet to be fully elucidated. The present study, based on bioinformatics analysis and experimental studies in vivo and in vitro, demonstrated that Hpa enhanced the crosstalk between TNBC cells and PLT to increase the supply of oxygen and nutrients, while also conferring tolerance of TNBC cells to oxygen and nutrient shortage, both of which are important for overcoming the stress of hypoxia and nutritional deprivation in the tumor microenvironment, thereby promoting malignant progression, including growth, angiogenesis and metastasis in TNBC. In addition, the hypoxia-inducible factor-1a (HIF-1a)/vascular endothelial growth factor-a (VEGF- a)/phosphorylated protein kinase B (p-)Akt axis may be the key pathway involved in the effects of Hpa on the biological processes mentioned above.
\end{abstract}

Correspondence to: Professor Guo-Wang Yang or Professor Gan-Lin Zhang, Department of Oncology, Beijing Hospital of Traditional Chinese Medicine, Capital Medical University, 23 Back Road of Art Gallery, Dongcheng, Beijing 100010, P.R. China

E-mail: yangguowang_bhtcm@126.com

E-mail: kalinezhang@163.com

Key words: triple-negative breast cancer, platelets, heparanase, hypoxia, metastasis
Therefore, improving local hypoxia, anti-Hpa treatment and inhibiting PLT activation may improve the prognosis of TNBC.

\section{Introduction}

Triple-negative breast cancer (TNBC) is a type of breast cancer that is estrogen receptor-negative (ER'), progesterone receptor-negative (PR) and human epidermal growth factor receptor 2-negative (HER2), and is characterized by high invasiveness and poor prognosis (1). A growing body of evidence suggests that TNBC therapy failure is mainly due to the inherently aggressive behavior of tumor cells and lack of recognized molecular targets for therapy (2). Thus, specific therapeutic targets for TNBC are urgent needed.

Platelets (PLT), which are mainly known for their role in hemostasis, also play a crucial role in nearly all stages of tumor progression (3). Various tumor cells have the ability to activate PLT to secrete a broad array of growth factors, including vascular endothelial growth factor (VEGF), PLT-derived growth factor (PDGF) and fibroblast growth factor (4-6), which are closely correlated with tumor growth, angiogenesis, metastasis and poor prognosis (7-14). However, whether there is crosstalk between PLT and TNBC cells that contributes to the progression of TNBC remains elusive.

Heparanase (Hpa), the only mammalian endoglycosidase that cleaves heparan sulfate, has been found to be upregulated in essentially all human tumors (15-18), contributing to tumor growth, angiogenesis, metastasis and poor prognosis (19-29). Hpa is abundantly expressed in both tumor cells and PLT, and it was previously confirmed that PLT and tumor Hpa are similar enzymes (30). In addition, concomitant upregulated expression of both Hpa and P-selectin (a recognized PLT activation marker) was found upon PLT activation (31). However, whether the Hpa from TNBC could activate PLT or enhance the crosstalk between TNBC and PLT remains unknown.

Almost all solid tumors, including TNBC, are constantly exposed to chronic or acute hypoxia (32-34). Hypoxia affects a variety of tumor-associated events, such as growth, angiogenesis and metastasis (35-38). In ovarian and pancreatic cancer, Hpa activity and the invasive ability of tumor cells were significantly enhanced by hypoxia, and this invasive ability may be blocked by specific Hpa-neutralizing antibodies (39). 
However, whether hypoxia can promote the progression of TNBC by enhancing the biological function of Hpa has not been fully elucidated.

The aim of the present study was to investigate the crosstalk between TNBC and PLT and its role in malignant progression. In addition, the role of Hpa and the effects of local conditions, such as hypoxia and nutritional deprivation, were explored.

\section{Materials and methods}

Exploring the correlation between Hpa and metastasis in TNBC from The Cancer Genome Atlas (TCGA) database. The RNA-Seq dataset of breast cancer and corresponding clinical information were downloaded from TCGA database and patients with TNBC (ER-, $\mathrm{PR}^{-}$and HER2-) were selected for further analysis.

In order to explore whether there is a statistically significant difference in the expression of Hpa between the metastasis and non-metastasis groups, the expression of Hpa was compared between the two groups.

Gene set enrichment analysis (GSEA). According to the median expression of Hpa, the samples of TNBC were divided into high and low Hpa expression groups.

GSEA was performed to explore the association between the expression of Hpa and 'BREAST CANCER METASTASIS UP'/'VEGF'/'VEGFR1/2' pathways. The number of permutations was set up to 1,000 and a false discovery rate (FDR) $<0.25$ was recognized as statistically significant.

Exploring the clinical value of Hpa. As the high expression of Hpa was significantly enriched in 'BREAST CANCER METASTASIS UP'/'VEGF'/'VEGFR1/2' pathways in TNBC, and all those pathways are associated with poor prognosis, we sought to compare the differences in disease-free survival (DFS) and overall survival (OS) between the high and low Hpa expression groups in TNBC.

Cell culture. The human TNBC cell lines MDA-MB-231-Hpa and MDA-MB-231-mock were gifts from Israel Vlodavsky, Cancer and Vascular Biology Research Center, Rappaport Faculty of Medicine, Technion (Haifa, Israel). The GFP inserts were manufactured in Glyconovo Technologies Co., Ltd. Human umbilical vascular endothelial cells (HUVECs) were purchased from the China Infrastructure of Cell Line Resource. All cells were cultured in DMEM (Gibco; Thermo Fisher Scientific, Inc.) supplemented with 10\% FBS (Sigma-Aldrich; Merck $\mathrm{KGaA})$ and $1 \%$ penicillin-streptomycin $(10,000 \mathrm{U} / \mathrm{ml}$ penicillin, 10,000 $\mu \mathrm{g} / \mathrm{ml}$ streptomycin; Gibco; Thermo Fisher Scientific, Inc.) in a humidified atmosphere $\left(5 \% \mathrm{CO}_{2}, 37^{\circ} \mathrm{C}\right)$. An oxygen-regulated incubator (Smartor118, Hua Yi Ning Chuang) was used to perform the study under hypoxia $\left(1 \% \mathrm{O}_{2}\right)$.

Animal care and establishment of nude mouse model in vivo. A total of 40 female BALB/c nude mice, aged 6-8 weeks and weighing 18-20 g, were obtained from the China Institute of Food and Drug Verification and Research. The animal experimental protocols were approved by the China Laboratory Animal Welfare and Animal Experimental Ethics Committee (2018030209). All the mice were maintained in specific pathogen-free barrier facilities at the Beijing Institute of Traditional Chinese Medicine, carefully bred, and humanely sacrificed by cervical dislocation at the study endpoint.

MDA-MB-231-Hpa-GFP and MDA-MB-231-mock-GFP cells in the logarithmic growth phase were harvested and counted, suspended in PBS, and the concentration was adjusted to $2 \times 10^{7} / \mathrm{ml}$ or $1.5 \times 10^{7} / \mathrm{ml}$. For the breast cancer model, $50 \mu 1$ cell suspension $\left(1 \times 10^{6}\right)$ was steadily injected into the fourth intramammary gland fat pad of each female $\mathrm{BALB} / \mathrm{c}$ nude mouse on either side at a uniform speed of $2 \mathrm{sec}$, named B-Hpa and B-mock. For the lung metastasis model, $100 \mu \mathrm{l}$ cell suspension $\left(1.5 \times 10^{6}\right)$ was steadily injected into the tail vein, named L-Hpa and L-mock. A normal group (no intervention) was set up at the same time. Each group included 8 mice.

In the breast cancer model, the tumor volume (V) was recorded twice per week and was calculated by the formula: $\mathrm{V}\left(\mathrm{mm}^{3}\right)=0.5 \times \mathrm{a} \mathrm{x} \mathrm{b}^{2}$ (a=shortest diameter, $\mathrm{b}=$ longest diameter).

For hematoxylin and eosin (H\&E) staining, the breast and lung tissues were fixed by immersion in $10 \%$ neutral buffered formalin at room temperature for $24 \mathrm{~h}$ and processed for paraffin embedding. The paraffin blocks of the tumor tissues were cut into $5-\mu \mathrm{m}$ sections and stained at room temperature with H\&E (10 min hematoxylin and 1 min eosin).

Exploring the expression of CD62P and VEGF-a in mouse serum. For ELISA, serum CD62P (cat. no. MPS00; R\&D Systems, Inc.) and VEGF-a (cat. no. MMV00; R\&D Systems, Inc.) were quantified using commercially available anti-mouse ELISA kits, according to the manufacturer's instructions. All assays were run in duplicate at a suitable dilution. The absorbance was read by a microplate reader (Multiskan GO, Thermo Fisher Scientific, Inc.) set at $450 \mathrm{~nm}$ and corrected at $540 \mathrm{~nm}$.

\section{In vivo exploration of the oxygen and nutrient supply inside} the solid tumor

Animal ultrasound imaging system. The Prospect animal ultrasound imaging system (S-Sharp Corporation) was used to measure the directional blood flow rate (Doppler mode), depicting the two-dimensional blood vessel distribution (Doppler mode) and quantitatively analyzing the local blood flow velocity (PW-mode), which may detect the blood supply in tumor tissue from multiple dimensions.

Immunofluorescence. The Hypoxyprobe ${ }^{\mathrm{TM}}$ Red APC Kit (cat. no. HP8-x) contains pimonidazole hydrochloride and Hypoxyprobe-1 Mab1 mouse monoclonal antibody, and it was used in the present study to detect hypoxia in breast tumors. One hour before the mice were sacrificed, each mouse received an intraperitoneal injection of $60 \mathrm{mg} / \mathrm{kg}$ pimonidazole hydrochloride (Hypoxyprobe ${ }^{\mathrm{TM}}$ Chemicon International; Thermo Fisher Scientific, Inc.). This compound was rapidly distributed to all tissues; however, pimonidazole forms adducts with thiol-containing proteins only at $\mathrm{pO}_{2} \leq 10 \mathrm{~mm} \mathrm{Hg}$. After $60 \mathrm{~min}$, according to the manufacturer's instructions (Chemicon International; Thermo Fisher Scientific, Inc.), the mice were sacrificed and the breast tumors were removed for further analyses. The hypoxic regions in the breast tumors were detected with the Hypoxyprobe-1 Mab1 mouse monoclonal antibody (1:50; cat. no. HP8-X; Chemicon International; Thermo Fisher Scientific, Inc.). 
Immunohistochemistry (IHC). Neovascularization and vasculogenic mimicry (VM) in the breast tumor and lung metastasis were identified by $\mathrm{CD}^{+} 1^{+} / \mathrm{PAS}^{+}$immunohistochemical staining. Endogenous peroxidase was inactivated with 3\% hydrogen peroxide at room temperature for $20 \mathrm{~min}$. Subsequently, the slides were soaked in $0.1 \mathrm{~mol} / 1$ citrate buffer $(\mathrm{pH} 6.0)$ and placed in an autoclave at $121^{\circ} \mathrm{C}$ for $2 \mathrm{~min}$ for antigen retrieval. After washing with PBS (pH 7.4), the sections were blocked with $1 \%$ BSA (cat. no. A8010; Solarbio) diluted in PBS at $37^{\circ} \mathrm{C}$ for $30 \mathrm{~min}$, incubated with anti-CD31 protein IgG (1:50; cat. no. ab28364; Abcam) at $4^{\circ} \mathrm{C}$ overnight, and again rinsed with PBS. The sections were then incubated with $0.5 \%$ PAS at room temperature for $10 \mathrm{~min}$ and washed with distilled water for 3 min. Finally, all the sections were counterstained with hematoxylin at room temperature for $5 \mathrm{~min}$.

\section{Preparation of the coculture supernatants}

Preparation of human PLT. The blood samples were collected from healthy volunteers who had not taken any drugs affecting PLT function and had not received other anticoagulant therapy within the 14 days preceding sample collection. PLT were prepared from the blood as previously described $(40,41)$ and finally suspended in DMEM $\left(1 \times 10^{8} / \mathrm{ml}\right)$.

Preparation of coculture supernatants. In order to study the role of the crosstalk between human PLT and TNBC cells in cancer progression, human PLT and MDA-MB231-Hpa-GFP/MDA-MB-231-mock-GFP cells were incubated together to obtain the coculture supernatants. Under physiological conditions, the lifespan of PLT is 7-10 days, whereas in vitro, this survival time was greatly shortened. Culture times of $30 \mathrm{~min}$ and $24 \mathrm{~h}$ in vitro were selected to mimic the early- and late-phase crosstalk in vivo, respectively. MDA-MB-231-Hpa-GFP/MDA-MB-231-mock-GFP cells at the logarithmic growth phase were harvested and counted, and the concentration was adjusted to $1 \times 10^{6} / \mathrm{ml}$ DMEM. A total of $1 \mu \mathrm{l}$ warmed $25 \mathrm{mM} \mathrm{CaCl}_{2}$ was added before the PLT suspensions $(1 \mathrm{ml})$ and the MDA-MB-231-Hpa-GFP/MDAMB-231-mock-GFP cell suspensions $(1 \mathrm{ml})$ were incubated together for $30 \mathrm{~min}$ or $24 \mathrm{~h}\left(37^{\circ} \mathrm{C}, 5 \% \mathrm{CO}_{2}\right)$ independently. After the indicated times, the coculture supernatants were centrifuged twice $(1,400 \mathrm{x}$ g for $20 \mathrm{~min}$, and then $13,000 \mathrm{x} \mathrm{g}$ for $2 \mathrm{~min}$ ) at $4^{\circ} \mathrm{C}$ to remove all the TNBC cells and PLT. PLT/MDA-MB-231-Hpa-GFP/MDA-MB-231-mockGFP cells were cultivated separately for $30 \mathrm{~min}$ or $24 \mathrm{~h}$ as controls independently, and the supernatants were stored at $-80^{\circ} \mathrm{C}$ until assayed.

\section{Identification of the supernatants}

Physical characteristics (concentration and size). The supernatants were analyzed by a nanoflow cytometer (N30 Nanoflow Analyzer, NanoFCM Inc.), which can sensitively and rapidly perform quantitative multiparameter analysis, surface protein profiling and sizing of individual extracellular vesicles (EVs) down to $40 \mathrm{~nm}$.

Angiogenesis-related factors. Following the manufacturer's instructions, the Bio-Plex Pro Human Cytokine 42-Plex Panel kit (Bio-Rad Laboratories, Inc.; cat. no. 12007238) and the Bio-Plex cytokines assay system (Bio-Plex 200, Bio-Rad
Laboratories, Inc.) were used to explore the genes of interest, and the results were analyzed by the Bio-Plex manager software, version 4.0 (Bio-Rad Laboratories, Inc.). The concentration of the respective cytokines was measured in $\mathrm{pg} / \mathrm{ml}$.

\section{Validating the biological functions of the supernatants} Proliferation analysis. Human PLT $\left(1 \times 10^{8} / \mathrm{ml}\right)$ and MDA-MB-231-Hpa-GFP/MDA-MB-231-mock-GFP cells $\left(1 \times 10^{6} / \mathrm{ml}\right)$ were cocultured. After the indicated times (30 $\mathrm{min} / 24 \mathrm{~h}$ ), the coculture supernatants were centrifuged twice $(1,400 \mathrm{xg}$ for $20 \mathrm{~min}$, and then $13,000 \mathrm{xg}$ for $2 \mathrm{~min})$ at $4^{\circ} \mathrm{C}$ to remove all the TNBCs and PLT. These supernatants with final concentrations of $20,40,60,80$ and $100 \%$ were used to interfere with the proliferation of the MDA-MB-231-Hpa-GFP and MDA-MB-231-mock-GFP cells. In addition, the GFP was recorded by BioTek Synergy H1 microplate reader (BioTek Instruments, Inc.) to reflect the proliferation indirectly.

Migration analysis. MDA-MB-231-Hpa-GFP and MDA-MB-231-mock-GFP cells were seeded into 6-well plates $\left(5 \times 10^{5}\right.$ cells/well) for $24 \mathrm{~h}$. After reaching a confluence of $100 \%$, the cell monolayers were scratched using a $200-\mu 1$ pipette tip and then the floating cells were removed by washing with PBS. Subsequently, the cells were incubated in DMEM with or without $20 \%$ supernatants $(30 \mathrm{~min} / 24 \mathrm{~h})$ and photographed every $8 \mathrm{~h}$. The wound healing rate was analyzed using Image J software 1.48 (National Institutes of Health).

Exploring the anti-stress potential of TNBC cells under conditions of stress (hypoxia and nutritional deprivation)

Proliferation assay. HUVECs, MDA-MB-231-Hpa-GFP and MDA-MB-231-mock-GFP cells were all seeded in 96-well plates at a density of $10^{3}$ cells/well with 10 replicates and cultured in a hypoxic environment $\left(1 \% \mathrm{O}_{2}, 5 \% \mathrm{CO}_{2}, 37^{\circ} \mathrm{C}\right)$ or normal environment $\left(20 \% \mathrm{O}_{2}, 5 \% \mathrm{CO}_{2}, 37^{\circ} \mathrm{C}\right)$. CCK-8 reagent $(10 \mu \mathrm{l})$ was added into each well at 24,48 and $72 \mathrm{~h}$, and the OD values at $450 \mathrm{~nm}$ were measured (Multiskan GO; Thermo Fisher Scientific, Inc.) $3 \mathrm{~h}$ later.

Colony formation assay. MDA-MB-231-Hpa-GFP and MDA-MB-231-mock-GFP cells were seeded into 6-well plates (500 cells/well) and cultured in a hypoxic environment $\left(1 \% \mathrm{O}_{2}, 5 \% \mathrm{CO}_{2}, 37^{\circ} \mathrm{C}\right)$ or normal environment $\left(20 \% \mathrm{O}_{2}\right.$, $5 \% \mathrm{CO}_{2}, 37^{\circ} \mathrm{C}$ ) for 7 days. The cells were fixed with $4 \%$ methanol and stained with $0.2 \%$ crystal violet solution for $30 \mathrm{~min}$ at room temperature, then washed with PBS 3 times. Finally, the number of colonies (minimum of 50 cells) was counted under an inverted microscope (TS100, Nikon Corporation) at a magnification of $\mathrm{x} 20$.

Capillary tube formation assay. Plates (96-well) were coated with Matrigel (50 $\mu 1 /$ well BD Biosciences), which was allowed to polymerize for $1 \mathrm{~h}$ at $37^{\circ} \mathrm{C}$. HUVECs, MDA-MB-231-Hpa-GFP and MDA-MB-231-mock-GFP cells at a density of $3 \times 10^{4} / 100 \mu 1$ were seeded into the wells coated with solid Matrigel, with or without the supernatants. The cells were then cultured under normal $\left(20 \% \mathrm{O}_{2}, 5 \% \mathrm{CO}_{2}, 37^{\circ} \mathrm{C}\right.$, $10 \% \mathrm{FBS}$ ) or stress (hypoxia and nutritional deprivation; $1 \% \mathrm{O}_{2}$, $5 \% \mathrm{CO}_{2}, 37^{\circ} \mathrm{C}, 0 \% \mathrm{FBS}$ ) conditions. The observation time for the HUVECs was $12 \mathrm{~h}$, and for MDA-MB-231-Hpa-GFP and 
MDA-MB-231-mock-GFP cells $24 \mathrm{~h}$. Images were captured using a Nikon camera (Nikon Corporation) at a magnification of $\mathrm{x} 4$ and then analyzed with AngioTool, version 0.5 (https://ccrod.cancer.gov/confluence/display/ROB2/Home).

\section{Exploring the key proteins and signaling pathways}

Protein extraction. MDA-MB-231-Hpa-GFP and MDA-MB-231-mock-GFP cells were cultured in normal $\left(20 \% \mathrm{O}_{2}, 5 \% \mathrm{CO}_{2}, 10 \% \mathrm{FBS}, 37^{\circ} \mathrm{C}\right)$, hypoxic $\left(1 \% \mathrm{O}_{2}, 5 \% \mathrm{CO}_{2}\right.$, $\left.10 \% \mathrm{FBS}, 37^{\circ} \mathrm{C}\right)$ and stress $\left(1 \% \mathrm{O}_{2}, 5 \% \mathrm{CO}_{2}, 0 \% \mathrm{FBS}, 37^{\circ} \mathrm{C}\right)$ environments, harvested at $24 \mathrm{~h}$, lysed in ice-cold RIPA buffer containing phosphatase and protease inhibitor (cat. no. R0020; Solarbio) for $30 \mathrm{~min}$, centrifuged for $10 \mathrm{~min}\left(12,000 \mathrm{x} \mathrm{g}, 4^{\circ} \mathrm{C}\right)$, and quantified by a BCA protein assay kit.

Western blot analysis. Equal amounts of proteins (30 $\mu \mathrm{g} / \mathrm{lane})$ were subjected to $10 \%$ SDS-PAGE and then electronically transferred onto a PVDF membrane (EMD Millipore). The membrane was blocked with TBS and 0.1\% Tween-20 (TBST) containing $10 \%$ non-fat milk at room temperature for $1 \mathrm{~h}$ and incubated overnight with the following antibodies at $4^{\circ} \mathrm{C}$ : Hpa1 (Abcam, cat. no. ab85543, 1:2,000), VEGF-a (Abcam, cat. no. ab46154, 1:1,000), hypoxia-inducible factor HIF-1a (Immunoway, cat. no. YT2133, 1:2,000), Akt (CST, 9272, 1:5,000), phosphorylated p-Akt (Cell Signaling Technology, Inc., cat. no. 9271, 1:1,000), and $\beta$-tubulin (Immunoway, YM3030, 1:10,000). After washing with TBST 3 times, the membrane was incubated in IgG secondary antibody conjugated to horseradish peroxidase (Abcam, cat. no. ab97040, 1:4,000) for $1 \mathrm{~h}$ at room temperature, washed with TBST again for $10 \mathrm{~min}$ at room temperature and exposed in a dark room.

Statistical analysis. Statistical data were evaluated using SPSS 19.0 (IBM Corp.) and GraphPad Prism 7 (GraphPad Software, Inc.). Data were analyzed using Student's t-test for two-group comparisons and one-way ANOVA followed by Bonferroni post hoc test for multiple comparisons if the collected data assumed normal distribution and equal variances. If the data did not assume normal distribution or equality of variances, Wilcoxon's rank sum test and Kruskal-Wallis ANOVA with ranks was used. The Kaplan-Meier method was used for survival analysis. $\mathrm{P}<0.05$ was considered to indicate a statistically significant difference.

\section{Results}

Hpa may act through VEGF-related pathways to promote angiogenesis and metastasis, and it may worsen the prognosis ofTNBC. A total of 100 patients with TNBC were retrieved from TCGA database. After 140 months of follow-up, 59 patients developed metastasis and 41 patients were non-metastatic. The expression of Hpa was significantly higher in the metastasis group compared with that in the non-metastasis group $(\mathrm{P}=0.0326)$. In addition, the survival analysis indicated that the high expression of Hpa was significantly associated with poor DFS $(\mathrm{P}<0.05)$ and OS $(\mathrm{P}<0.05$; Fig. 1A).

Based on the median expression of Hpa, the 100 TNBC patients were divided into the high $(n=50)$ and the low $(n=50)$ Hpa expression groups. GSEA analysis indicated that the high expression of Hpa was significantly enriched in 'BREAST
CANCER METASTASIS UP', 'VEGF' and 'VEGFR1/2' pathways (Fig. 1B).

Hpa promotes PLT activation in a TNBC mouse model. ELISA of the plasma CD62P revealed that, in the breast cancer model, the CD62P level was significantly higher in the B-Hpa compared with that in the $\mathrm{B}-$ mock $(\mathrm{P}=0.0002)$ and normal groups $(\mathrm{P}=0.0281)$, and significantly higher in the $\mathrm{B}$-mock compared with that in the normal group ( $\mathrm{P}=0.0002$; Fig. 2A). In the lung metastasis model, the $\mathrm{CD} 62 \mathrm{P}$ level was significantly higher in the L-Hpa $(\mathrm{P}=0.0002)$ and L-mock groups $(\mathrm{P}=0.0011)$ compared with that in the normal group (Fig. 2B). These results indicated the PLT activation was higher in TNBC-bearing mice compared with that in normal mice and, to some extent, was further enhanced by Hpa.

Hpa promotes blood supply, growth and metastasis in a TNBC mouse model. Hpa was shown to promote blood supply in the TNBC mouse model.

General observation. In the breast tumor group, there was a small amount of dried blood (scab) on the surface of the breast tumors in the B-Hpa group even on the 28th day after tumor cell transplantation. However, these scabs were more prominent in the B-mock group (Fig. 3A). In the lung metastasis model, the dark red area of lung tissue, which reflects inadequate blood supply, was only observed in the L-mock group, and the size of each metastatic lesion was larger compared with the B-mock group (Fig. 3A).

Ultrasonic examination. The internal blood supply of breast tumors was significantly enriched in the B-Hpa group compared with that in the B-mock group (Fig. 3B). In addition, based on a large number of anatomical observations, the blood supply of the breast tumors relies on two major vessels, and the flow through these major vessels was significantly enriched in the B-Hpa compared with that in the B-mock group (Fig. 3B).

HP8 IHC. All the hypoxic regions were observed at the tumor margin, and these hypoxic regions were significantly larger in the B-mock compared with those in the B-Hpa group (Fig. 3C).

PAS/CD31 IHC. In the breast tumor and lung metastases (confirmed by H\&E; Fig. 3D), the extent of angiogenesis $\left(\mathrm{CD} 31^{+}\right)$and $\mathrm{VM}\left(\mathrm{PAS}^{+} \mathrm{CD} 31^{-}\right)$, which contribute to the blood supply of the tumor, was markedly greater in the $\mathrm{B} / \mathrm{L}-\mathrm{Hpa}$ (small and dense VM) compared with that in the B/L-mock group (large and sparse VM) (Fig. 3E).

Angiogenesis-related factors. The expression trend of VEGF-a in the experimental nude mouse plasma was consistent with the expression of CD62P (Fig. 3F).

Hpa promotes tumor growth in a TNBC mouse model. The volume of the breast tumors was significantly larger in the B-Hpa compared with that in the B-mock group, and the statistical significance of the difference was further evident with tumor growth. The breast tumor net weights (removed skin and blood scab) were significantly higher in the B-Hpa compared with those in the B-mock group (Fig. 3G). 
A

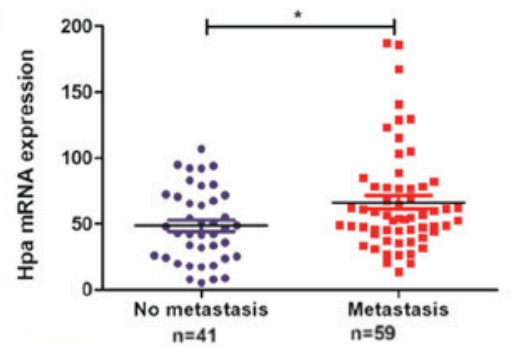

B Breast cancer metastasis up pathway

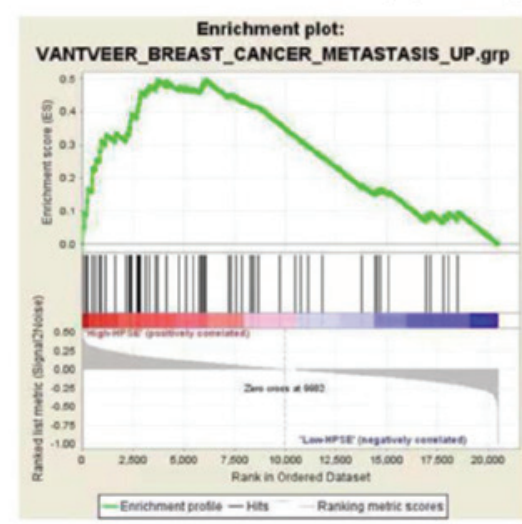

High Hpa

expression

Low Hpa
expression

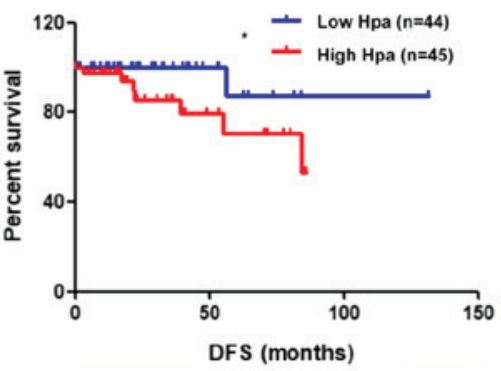

VEGF pathway

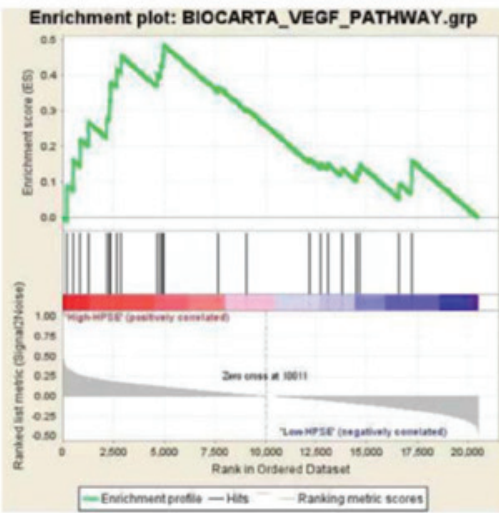

High Hpa

expression

Figure 1. Association of the expression of Hpa with metastasis, survival and relevant pathways in TNBC, based on TCGA database. (A) Comparison of Hpa expression between the metastasis and non-metastasis groups in TNBC. Survival analysis (DFS and OS) between the high and low Hpa expression groups ${ }^{*} \mathrm{P}<0.05$ indicated statistical significance. (B) Exploring the pathways which related to high Hpa expression by GSEA in TNBC. Hpa, heparanase; TNBC, triple-negative breast cancer; VEGF, vascular endothelial growth factor; TCGA, The Cancer Genome Atlas; DFS, disease-free survival; OS, overall survival; GSEA, Gene Set Enrichment Analysis.
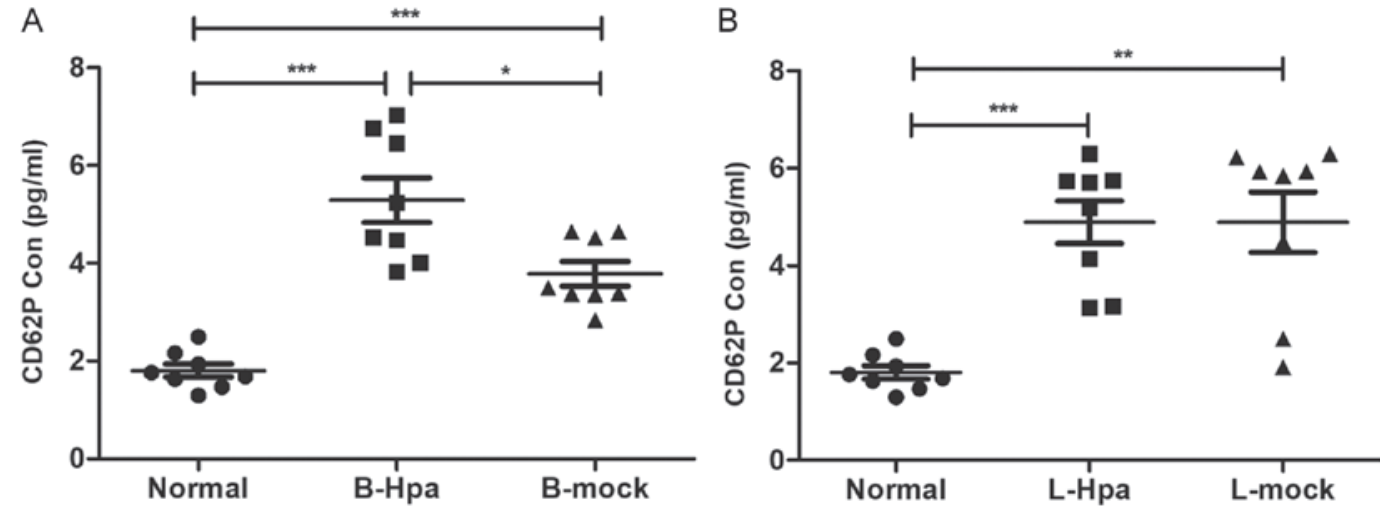

Figure 2. Analysis of plasma CD62P expression by ELISA. (A) Plasma CD62P expression in the B-Hpa, B-mock and normal groups. $\mathrm{P}<0.05$ indicated statistical significance. (B) Plasma CD62P expression in the L-Hpa, L-mock and normal groups. P<0.05 indicated statistical significance. B-Hpa group, MDA-MB-231-Hpa-GFP breast tumor group; B-mock group, MDA-MB-231-mock-GFP breast tumor group; L-Hpa group, MDA-MB-231-Hpa-GFP lung metastasis group; L-mock group, MDA-MB-231-mock-GFP lung metastasis group. Hpa, heparanase. ${ }^{*} \mathrm{P}<0.05,{ }^{* * *} \mathrm{P}<0.005$ and ${ }^{* * * *} \mathrm{P}<0.0005$.

Hpa promotes metastasis in a TNBC mouse model. The weights of the lung metastases were significantly higher in the L-Hpa compared with those in the L-mock group (Fig. 3G).

The intimate crosstalk between TNBC and PLT promotes bioactive substance release and is enhanced by Hpa. The physical characteristics of the supernatants (Fig. 4A; Table I) were as follows: i) Within a certain period of time (30 min-24 h), prolonging the culture time promoted release of bioactive substances: Hpa + PLT $(24 \mathrm{~h})>\mathrm{Hpa}+\mathrm{PLT}$ (30 min); mock + PLT $(24 \mathrm{~h})>$ mock + PLT (30 min); Hpa (24 h) >Hpa (30 min); mock (24 h) >mock (30 min); PLT (24 h) >PLT (30 min). ii) Coculture promoted release of bioactive substances: Hpa + PLT (30 min) >Hpa (30 min); Hpa + PLT (30 min) >PLT (30 min); mock + PLT (30 min) >mock (30 min); mock + PLT (30 min) >PLT (30 min); Hpa + PLT (24 h) >Hpa (24 h); Hpa + PLT (24 h) >PLT (24 h); mock + PLT (24 h) >mock (24 h); mock + PLT (24 h) >PLT (24 h). iii) The 


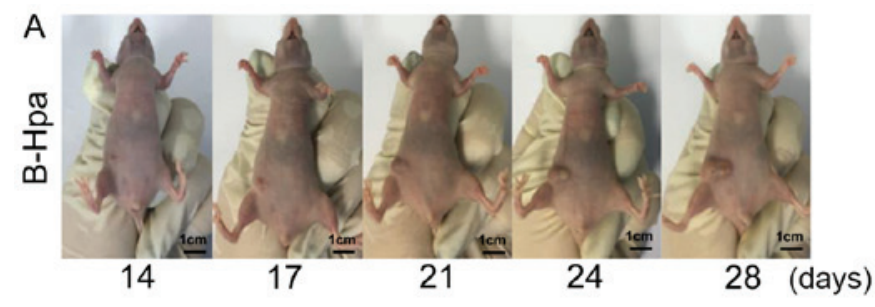

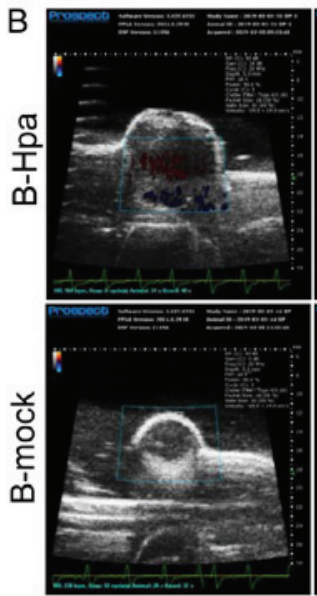

Internal blood supply

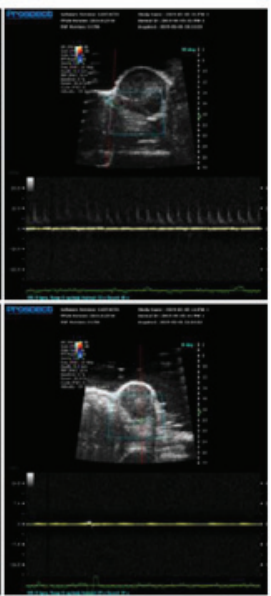

Blood flow in the major vessels

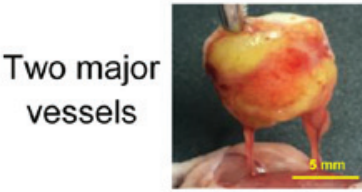

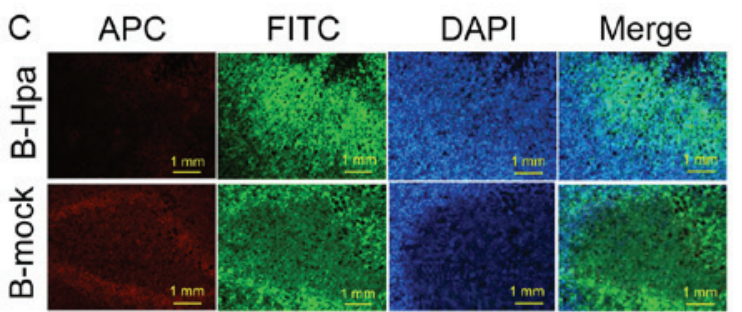
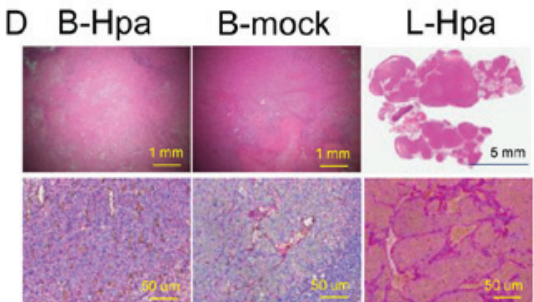

L-mock
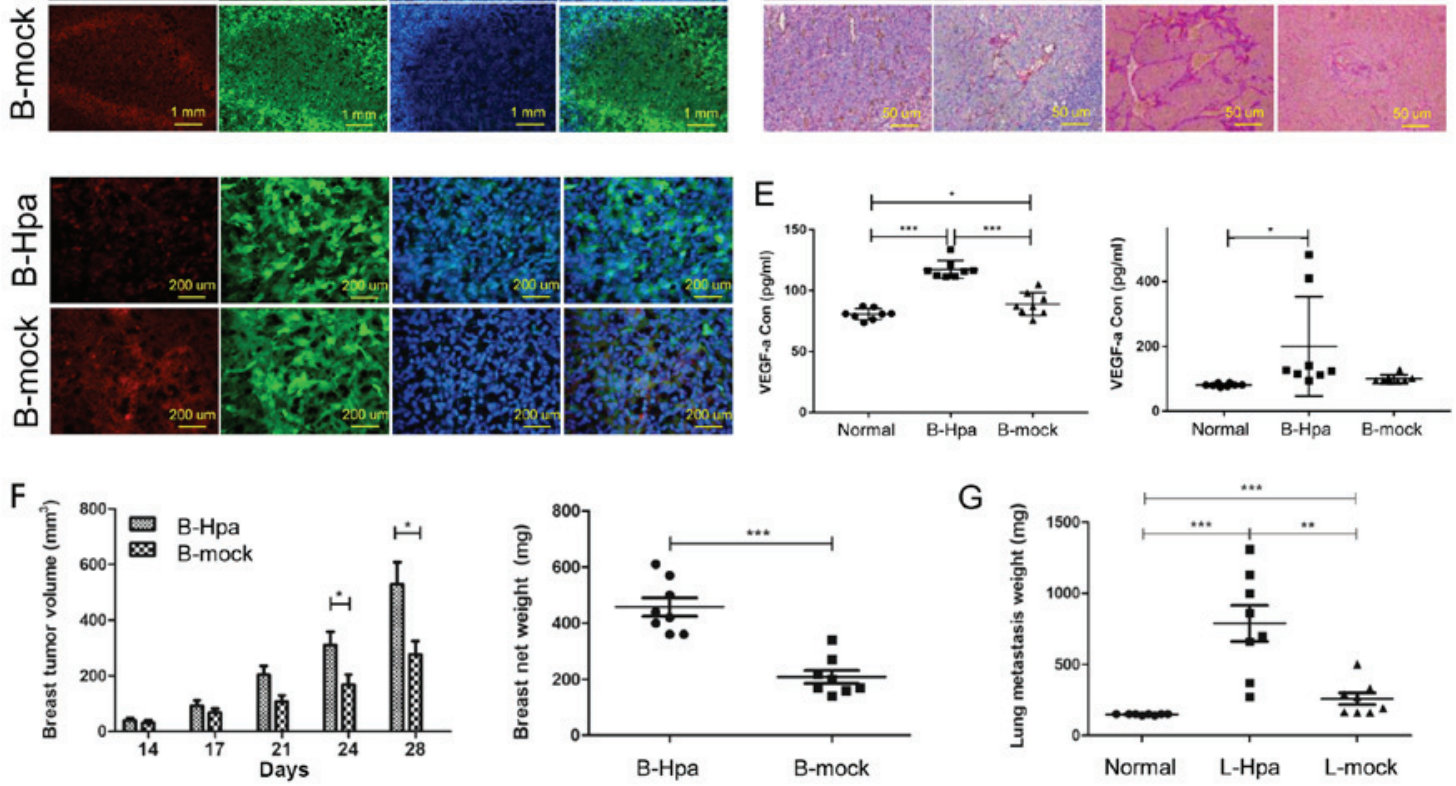

Figure 3. Blood supply within the solid tumor. (A) The general appearance of the breast tumors was recorded from the 14 th to the 28 th day after the tumor cells were injected in the B-Hpa and B-mock group. The lung metastases of the L-Hpa and L-mock group were recorded after terminating the in vivo test. (B) The breast tumor relies on two major vessels for blood supply. The animal ultrasound imaging system was used to compare the internal blood supply and the blood flow in the major vessels between the B-Hpa and B-mock groups. (C) Hypoxic region in B-Hpa and B-mock breast tumors were detected with a Hypoxyprobe $^{\mathrm{TM}}$ Red APC Kit. (D) Hematoxylin and eosin staining of the B-Hpa, B-mock, L-Hpa and L-mock tumors. (E) PAS/CD31 staining of the B-Hpa, B-mock, L-Hpa and L-mock tumors. (F) ELISA of the plasma CD62P expression in the breast tumor model and the lung metastasis model. P $<0.05$ indicated statistical significance. (G) Breast tumor volume and net weight of the breast tumor models in the B-Hpa and B-mock groups. P<0.05 indicated statistical significance. Lung metastasis weight of the lung metastasis models in the L-Hpa and L-mock groups. P $<0.05$ indicated statistical significance. B-Hpa group, MDA-MB-231-Hpa-GFP breast tumor group; B-mock group, MDA-MB-231-mock-GFP breast tumor group; L-Hpa group, MDA-MB-231-Hpa-GFP lung metastasis group; L-mock group, MDA-MB-231-mock-GFP lung metastasis group. VEGF, vascular epithelial growth factor; Hpa, heparanase. * $<0.05$, ${ }^{* *} \mathrm{P}<0.005$, and ${ }^{* * *} \mathrm{P}<0.0005$.

ability of promoting bioactive substance release was enhanced by Hpa: Hpa + PLT (30 min) >mock + PLT (30 min); Hpa +
PLT $(24$ h) $>$ mock + PLT (24 h). iv) There was no statistically significant difference in the size of the EVs in the supernatants. 
A

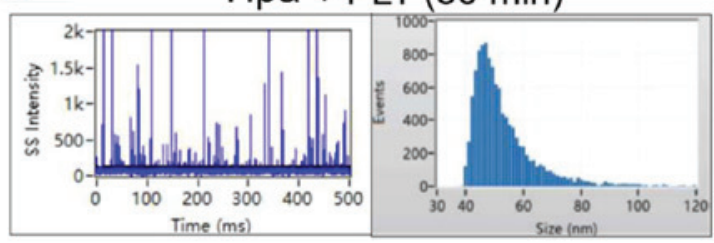

mock + PLT (30 min)

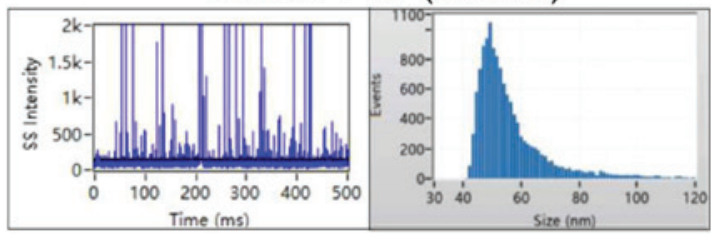

PLT (30 min)

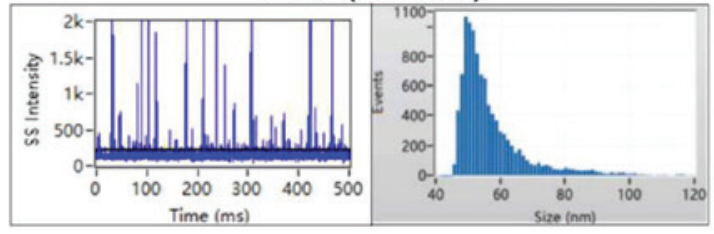

Hpa(30 min)

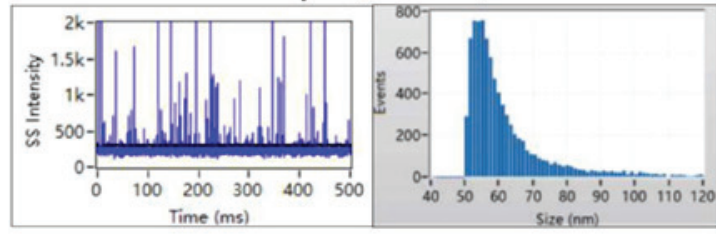

mock (30 min)

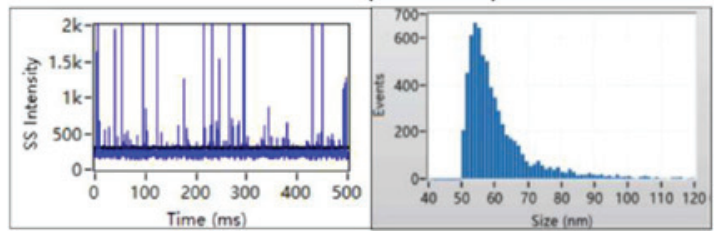

Hpa + PLT (24 h)

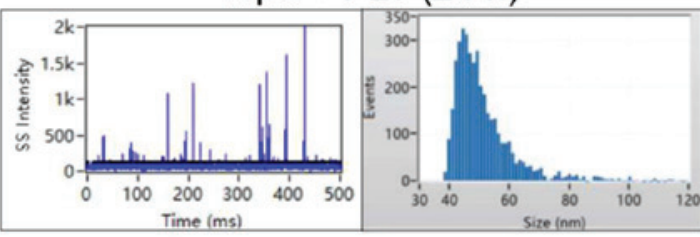

mock + PLT (24 h)

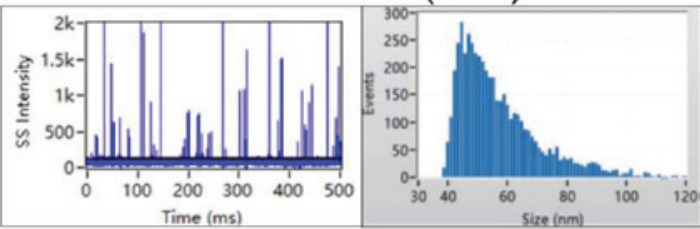

PLT (24 h)

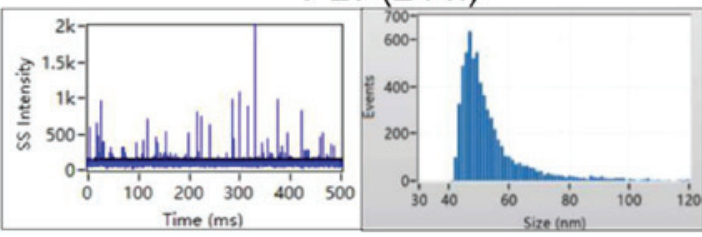

$\operatorname{Hpa}(24 \mathrm{~h})$

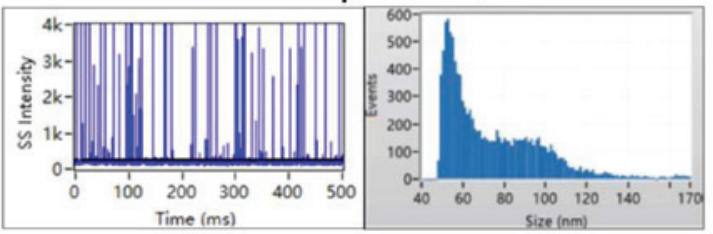

mock $(24 \mathrm{~h})$

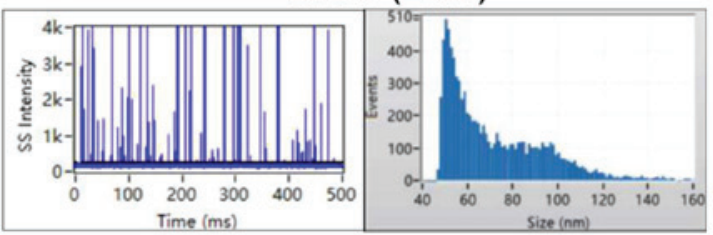

B

Expression of VEGF-a in the supernatants

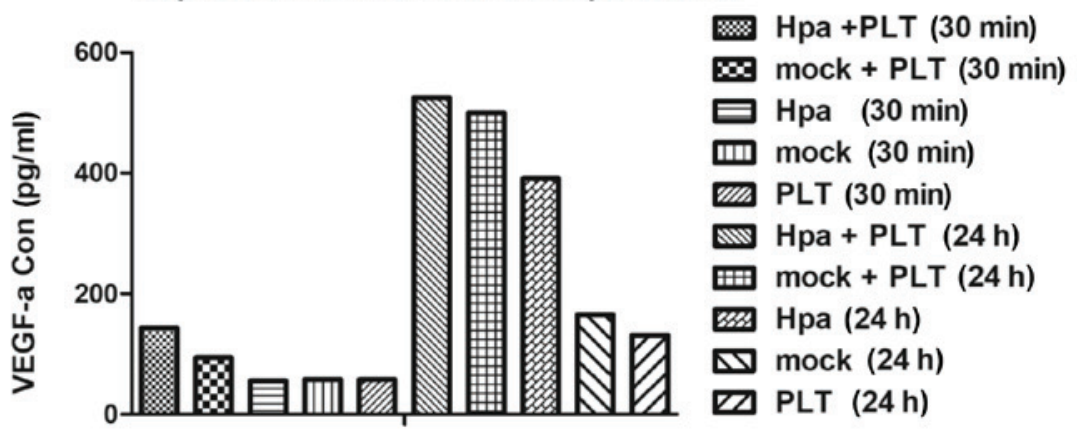

Figure 4. Identification of the coculture supernatants. (A) Physical characteristics (concentration and size) of the supernatants. (B) Expression of VEGF-a in the supernatants. Hpa + PLT (30 min), coculture of MDA-MB-231-Hpa-GFP cells and platelets for $30 \mathrm{~min}$; Hpa + PLT (24 h), coculture of MDA-MB-231-Hpa-GFP cells and platelets for $24 \mathrm{~h}$; mock + PLT (30 min), coculture of MDA-MB-231-mock-GFP cells and platelets for 30 min; mock + PLT (24 h), coculture of MDA-MB-231-Hpa-GFP cells and platelets for $24 \mathrm{~h}$; PLT (30 min), platelet culture for $30 \mathrm{~min}$; PLT (24 h), platelet culture for $24 \mathrm{~h}$; Hpa (30 min), MDA-MB-231-Hpa-GFP cell culture for $30 \mathrm{~min}$; Hpa (24 h), MDA-MB-231-Hpa-GFP cell culture for $24 \mathrm{~h}$; mock (30 min), MDA-MB-231-mock-GFP cell culture for $30 \mathrm{~min}$; mock (24 h), MDA-MB-231-mock-GFP cell culture for $24 \mathrm{~h}$. Hpa, heparanase; VEGF, vascular epithelial growth factor; PLT, platelets.

As regards the angiogenesis-related factors of the supernatants, the expression trend of VEGF-a in the supernatants was consistent with the physical characteristics (Fig. 4B).
The bioactive substances released by the intimate crosstalk between TNBC and PLT promote the proliferation and migration of TNBC cells, and these effects are enhanced by $\mathrm{Hpa}$ 
Table I. Physical characteristics of the supernatants.

\begin{tabular}{llc}
\hline Sample (time) & Size $(\mathrm{nm})$ & Concentration \\
\hline Hpa + PLT (30 min) & $49.28 \pm 11.05$ & $5.01 \times 10^{11}$ \\
mock + PLT (30 min) & $52.84 \pm 12.66$ & $1.15 \times 10^{11}$ \\
Hpa (30 min) & $57.59 \pm 12.32$ & $3.71 \times 10^{9}$ \\
mock (30 min) & $57.59 \pm 9.24$ & $2.87 \times 10^{9}$ \\
PLT (30 min) & $54.03 \pm 11.6$ & $9.2 \times 10^{9}$ \\
Hpa + PLT (24 h) & $49.09 \pm 10.36$ & $1.7 \times 10^{12}$ \\
mock + PLT (24 h) & $52.84 \pm 14.9$ & $1.11 \times 10^{12}$ \\
Hpa (24 h) & $65.91 \pm 23.46$ & $5.13 \times 10^{9}$ \\
mock (24 h) & $64.72 \pm 22.77$ & $4.04 \times 10^{9}$ \\
PLT (24 h) & $49.28 \pm 12.11$ & $2.81 \times 10^{10}$
\end{tabular}

$\mathrm{Hpa}+\mathrm{PLT}$ (30 min), coculture of MDA-MB-231-Hpa-GFP cells and platelets for $30 \mathrm{~min}$; mock + PLT (30 min), coculture of MDA-MB-231-mock-GFP cells and platelets for $30 \mathrm{~min}$; Hpa (30 min), MDA-MB-231-Hpa-GFP cell culture for $30 \mathrm{~min}$; mock (30 min), MDA-MB-231-mock-GFP cell culture for $30 \mathrm{~min}$; PLT (30 min), platelet culture for $30 \mathrm{~min}$; Hpa + PLT (24 h), coculture of MDA-MB-231-Hpa-GFP cells and platelets for $24 \mathrm{~h}$; mock + PLT (24 h), coculture of MDA-MB-231-Hpa-GFP cells and platelets for $24 \mathrm{~h}$; Hpa (24 h), MDA-MB-231-Hpa-GFP cell culture for $24 \mathrm{~h}$; mock (24 h), MDA-MB-231-mock-GFP cell culture for $24 \mathrm{~h}$; PLT $(24 \mathrm{~h})$, platelet culture for $24 \mathrm{~h}$.

Proliferation analysis. The GFP fluorescence of the MDA-MB-231-Hpa-GFP and MDA-MB-231-mock-GFP cells were tracked every $24 \mathrm{~h}$ for $120 \mathrm{~h}$. It was observed that the supernatants (coculture of MDA-MB-231-Hpa-GFP cells and PLT for $30 \mathrm{~min} /$ coculture of MDA-MB-231-mock-GFP cells and PLT for $30 \mathrm{~min}$ ) promoted cell proliferation individually in a concentration- and time-dependent manner: $100 \% \mathrm{Hpa}$ + PLT $(30 \mathrm{~min})>80 \% \mathrm{Hpa}+\mathrm{PLT}(30 \mathrm{~min})>60 \% \mathrm{Hpa}+$ PLT $(30 \mathrm{~min})>40 \% \mathrm{Hpa}+\mathrm{PLT}(30 \mathrm{~min})>20 \% \mathrm{Hpa}+\mathrm{PLT}$ (30 $\mathrm{min})>$ control; $100 \%$ mock + PLT $(30 \mathrm{~min})>80 \%$ mock + PLT $(30 \mathrm{~min})>60 \%$ mock + PLT $(30 \mathrm{~min})>40 \%$ mock + PLT $(30 \mathrm{~min})>20 \%$ mock + PLT $(30 \mathrm{~min})>$ control. As the proliferation ability of the cells treated with supernatants at a concentration of $20 \%$ exhibited a statistically significant difference compared with the control group, the final concentration of $20 \%$ was used in the subsequent experiments. In addition, the proliferation ability of the cells cultured with supernatant for $24 \mathrm{~h}$ was significantly higher compared with that of cells cultured with supernatant for $30 \mathrm{~min}$ : Hpa + PLT (24 h) >Hpa + PLT (30 min); mock + PLT (24 h) > mock + PLT (30 min) (Fig. 5A).

Migration analysis. The supernatants of the coculture of MDA-MB-231-Hpa-GFP cells and PLT or MDA-MB-231-mock-GFP cells and PLT promoted the migration of the cells individually: Hpa + PLT (30 min) $>$ Hpa-control, Hpa + PLT (24 h) >Hpa-control, mock + PLT (30 min) >mock-control, mock + PLT (24 h) >mock-control; $\mathrm{P}=0.0007,0.0007,0.001$ and 0.0056 , respectively. The migration ability of the cells cultured with supernatant for $24 \mathrm{~h}$ was significantly higher compared with that of cells cultured for
$30 \mathrm{~min}$, and this effect was enhanced by Hpa: Hpa + PLT (24 h) >Hpa + PLT (30 min), mock + PLT (24 h) >mock + PLT (30 min); P=0.002 and 0.0185, respectively; Hpa + PLT $(24 \mathrm{~h})>$ mock + PLT (24 h), Hpa + PLT (30 min) >mock + PLT (30 min); $\mathrm{P}=0.0166$ and 0.008 , respectively (Fig. 5B).

Hpa contributes to TNBC cells tolerating and overcoming hypoxia and stress environment

Proliferation analysis. The proliferation ability of TNBC cells and HUVECs were enhanced under hypoxic conditions, and the proliferation ability of TNBC cells was further enhanced by Hpa. However, the difference in the proliferation ability did not reach statistical significance (Fig. 6A).

Colony formation assay. Hypoxia significantly promoted the colony-forming ability of TNBC cells: Hpa-hypoxia $>$ Hpa-normal, mock-hypoxia >mock-normal; Hpa significantly promoted thecolony-formingability ofTNBCcells:Hpa-hypoxia $>$ mock-hypoxia, Hpa-normal >mock-normal; hypoxia further enhanced the ability of Hpa to promote the colony-forming ability of TNBC cells: (Hpa-hypoxia-Hpa-normal)/Hpa-normal $>$ (mock-hypoxia-mock-normal)/mock-normal (Fig. 6B).

Capillary tube formation assay. HUVECs: In a stress environment, there was a significantly decreased integrity of the vascular structure (evaluated by total number of junctions) compared with a normal environment. However, the supernatants $(\mathrm{Hpa}+\mathrm{PLT} / \mathrm{mock}+\mathrm{PLT})$ significantly increased the vascular structure formation by HUVECs, and these effects were further enhanced by Hpa (Fig. 6C). TNBC cells: In a stress environment, MDA-MB-231-Hpa-GFP cells exhibited a significantly increased VM compared with a normal environment. However, the VM of MDA-MB-231-mock-GFP cells was significantly decreased compared with a normal environment. Under normal conditions, there was no difference in VM between MDA-MB-231-Hpa-GFP and MDA-MB-231-Hpa-GFP cells. However, under stress conditions, MDA-MB-231-Hpa-GFP cells exhibited a significantly increased VM compared with MDA-MB-231-mock-GFP cells (Fig. 6D).

Hpa protein expression and its ability to promote growth, angiogenesis and metastasis may be enhanced through the HIF-1a/VEGF-a/p-AKT pathway, which is induced by hypoxia, and finally overcome hypoxia and stress conditions to promote tumor progression in TNBC. HIF-1 $\alpha$, the expression of which is induced by hypoxia, is a critical responsive element to hypoxia. Hpa and VEGF-a are target genes of HIF-1 $\alpha$, and they are both gradually upregulated under hypoxic and stress conditions. Furthermore, p-AKT activation was observed at the same time (Fig. 7).

\section{Discussion}

A growing body of evidence suggests that failure in TNBC treatment is mainly due to the inherently aggressive behavior of tumor cells and the lack of recognized molecular targets for therapy (2). An increasing number of studies have indicated that Hpa plays an important role in tumor growth, 
A

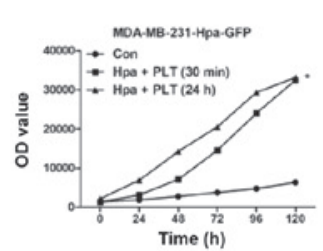

B
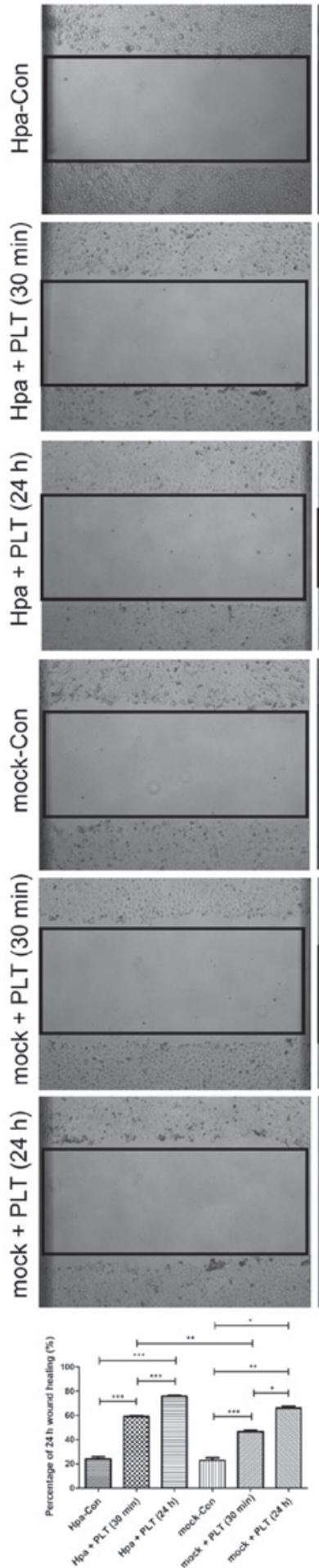

MOA-MB.231-mock-GFP
- COn

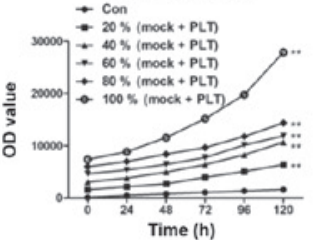

$8 \mathrm{~h}$
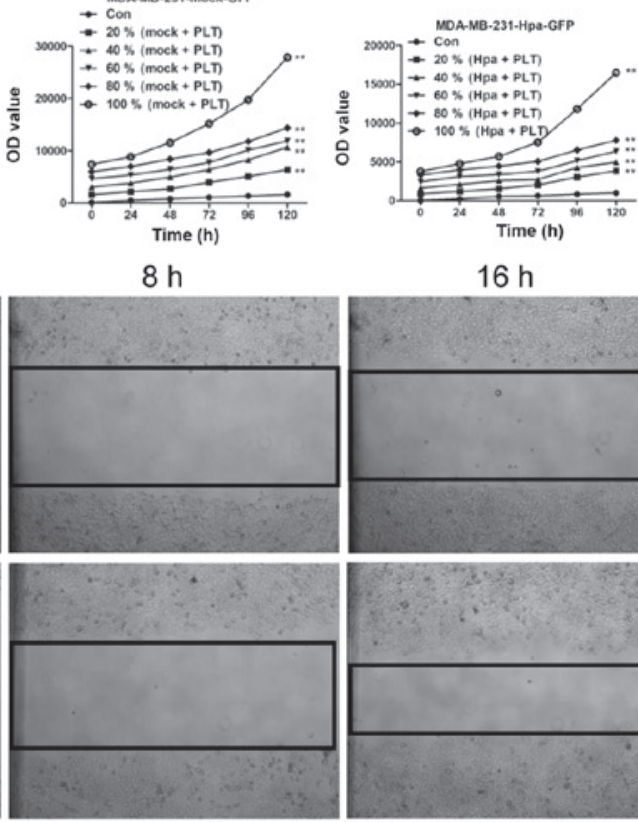

$16 \mathrm{~h}$
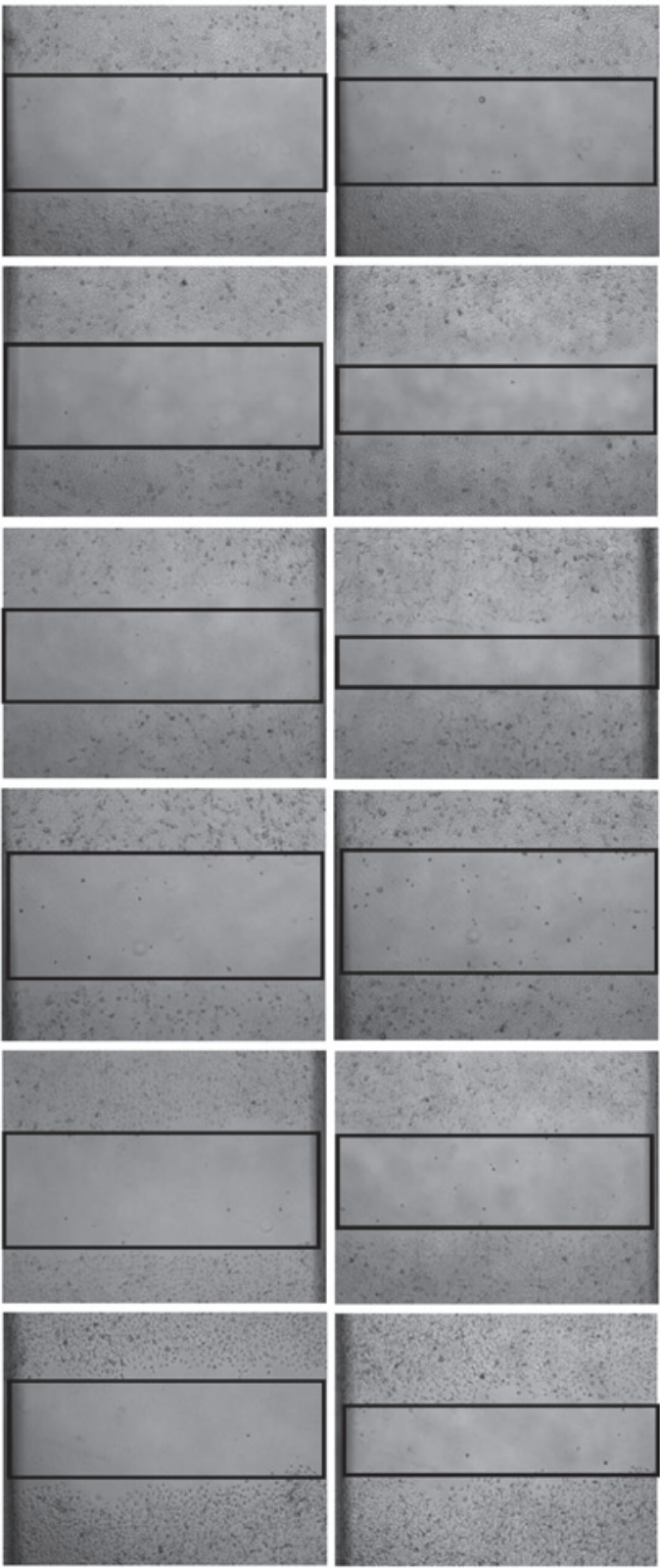

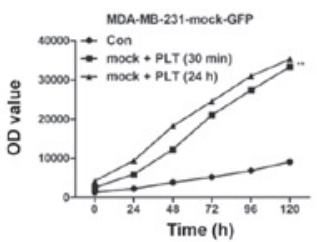

$24 \mathrm{~h}$
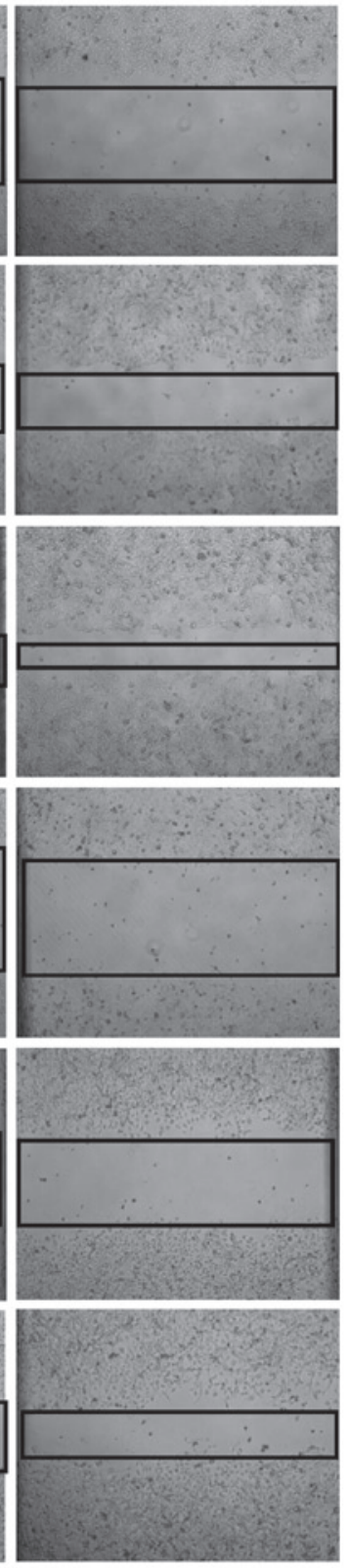

Figure 5. Biological functions of the supernatants. (A) Validating the proliferation-promoting function of the supernatants (MDA-MB-231-Hpa-GFP cell and platelet coculture for $30 \mathrm{~min} / \mathrm{MDA}-\mathrm{MB}-231$-mock-GFP cell and platelet coculture for $30 \mathrm{~min}$ ) by proliferation analysis. (B) Validating the migration-promoting function of the supernatants (MDA-MB-231-Hpa-GFP cell and platelet coculture for $30 \mathrm{~min} / \mathrm{MDA}-\mathrm{MB}-231-\mathrm{mock}-\mathrm{GFP}$ cell and platelet coculture for $30 \mathrm{~min}$ ) by migration analysis. Hpa + PLT, coculture of MDA-MB-231-Hpa-GFP cells and platelets for $30 \mathrm{~min}$; mock + PLT, coculture of MDA-MB-231-mock-GFP cells and platelets for $30 \mathrm{~min}$; Hpa + PLT (30 min), coculture of MDA-MB-231-Hpa-GFP cells and platelets for $30 \mathrm{~min}$; Hpa + PLT (24 h), coculture of MDA-MB-231-Hpa-GFP cells and platelets for $24 \mathrm{~h}$; mock + PLT (30 min), coculture of MDA-MB-231-mock-GFP cells and platelets for $30 \mathrm{~min}$; mock + PLT (24 h), coculture of MDA-MB-231-Hpa-GFP cells and platelets for $24 \mathrm{~h}$. Hpa, heparanase; PLT, platelets. ${ }^{*} \mathrm{P}<0.05,{ }^{* *} \mathrm{P}<0.005$, and ${ }^{* * *} \mathrm{P}<0.0005$. 
A
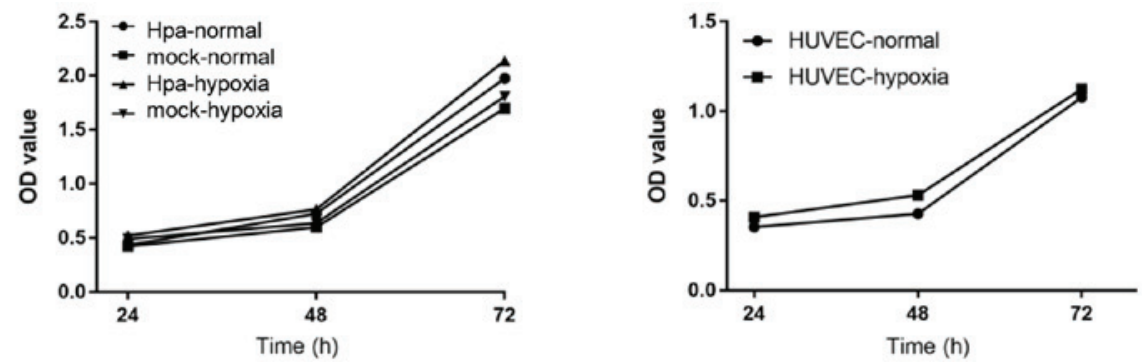

B
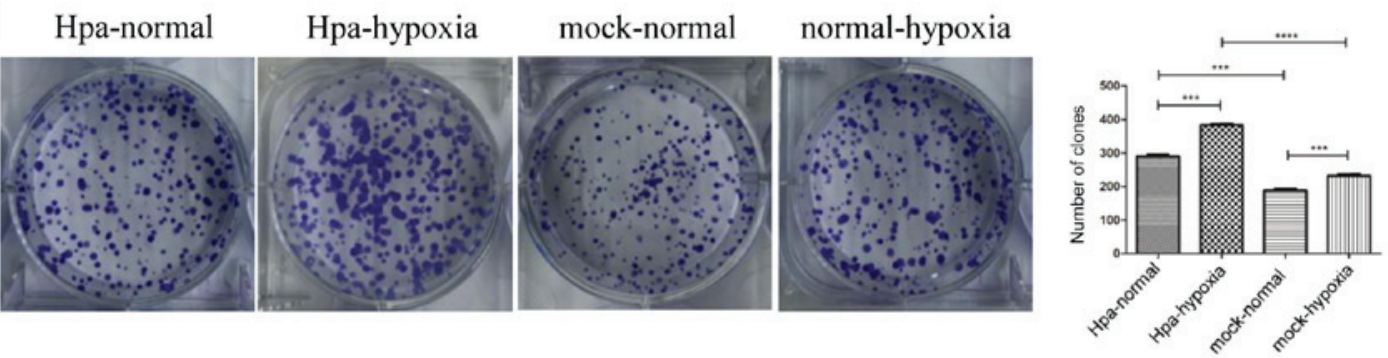

$\mathrm{C}$
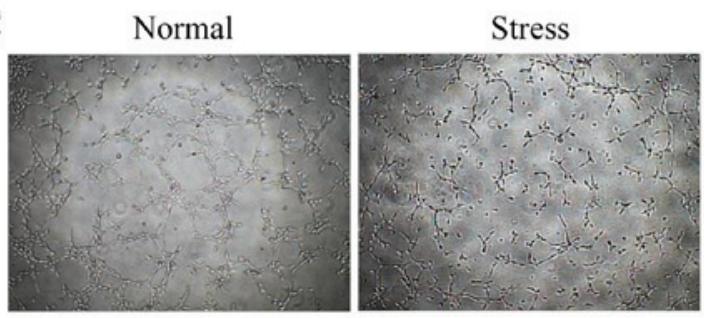

Stress $(\mathrm{Hpa}+\mathrm{PLT})$

Stress (mock + PLT)
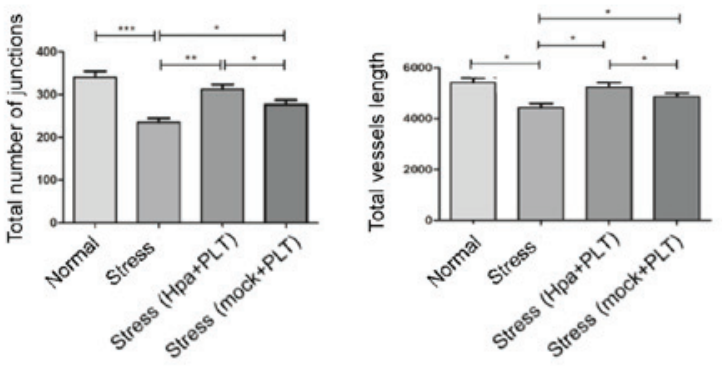

$\mathrm{D}$

Hpa-normal

Hpa-stress
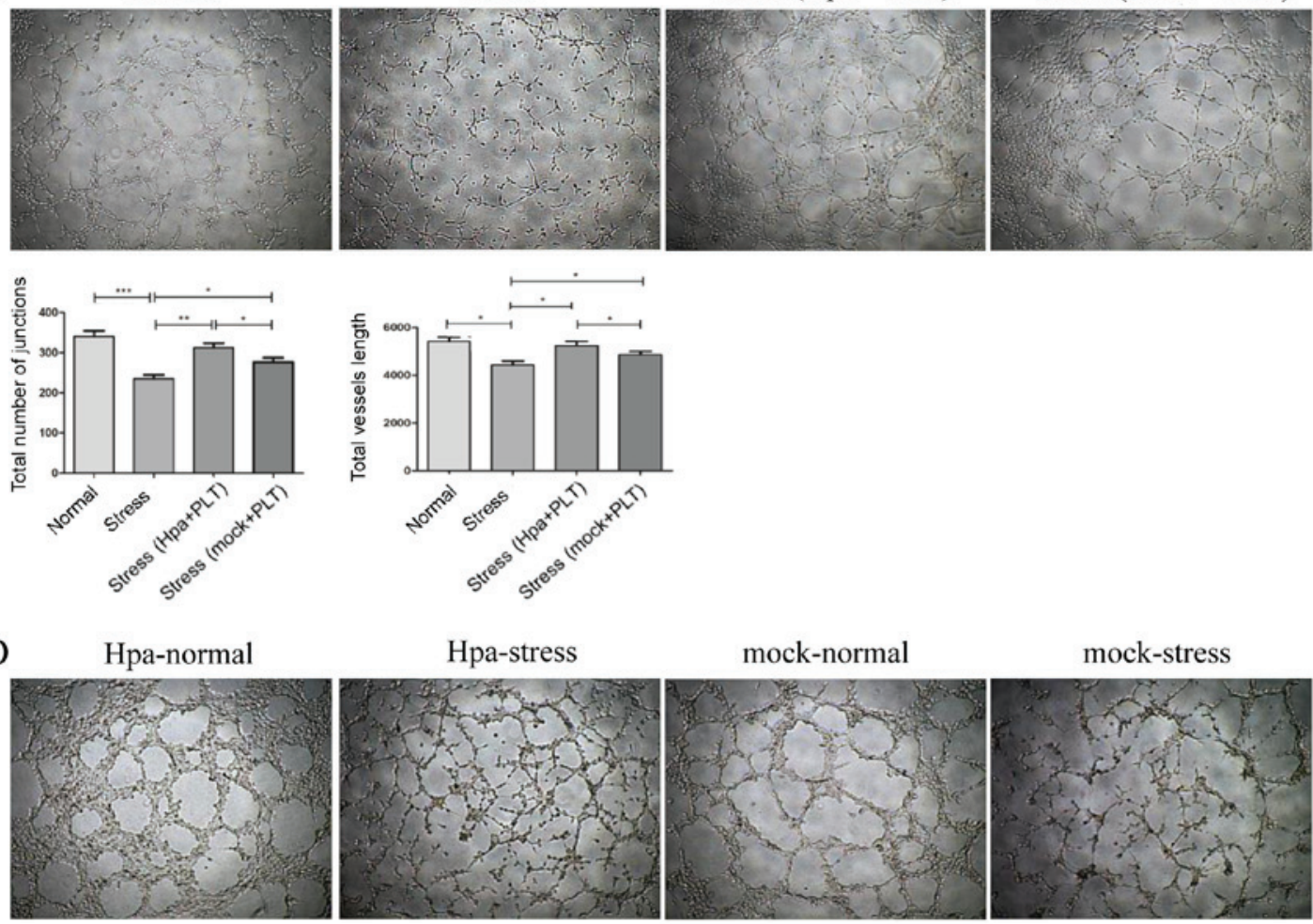

mock-normal

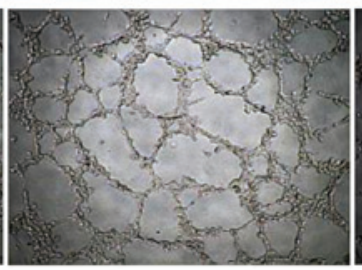

mock-stress
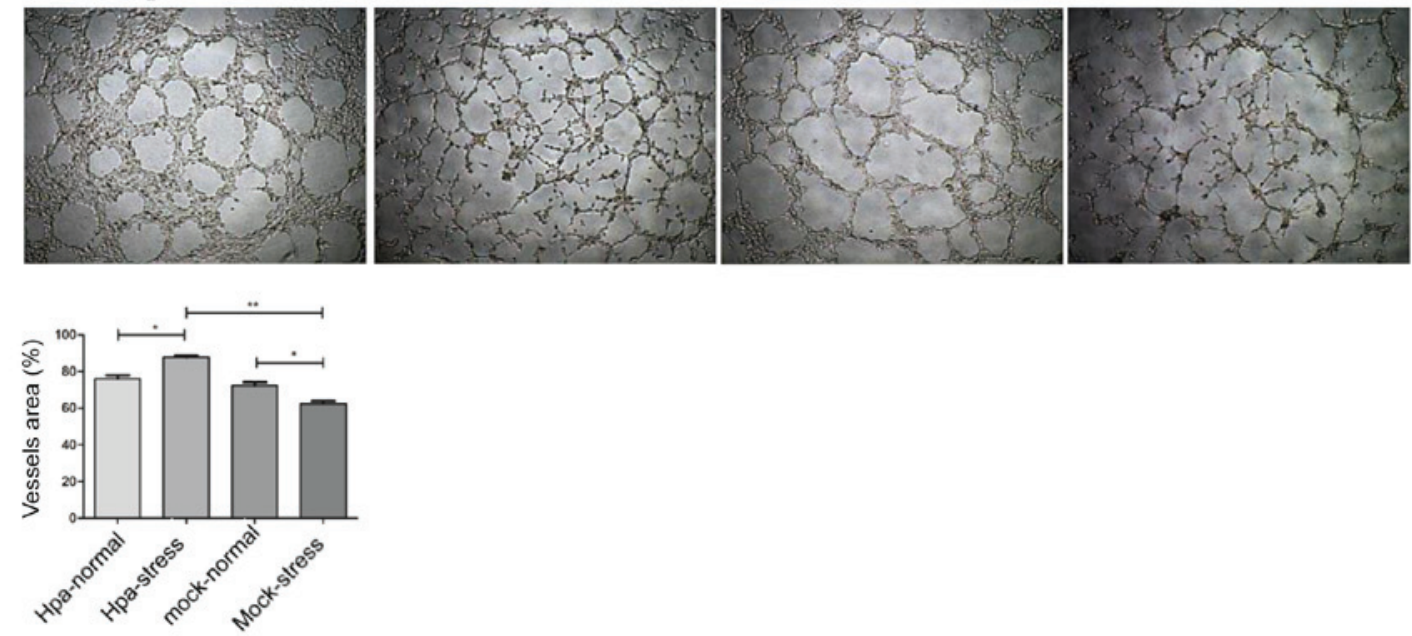

Figure 6. Anti-stress potential of TNBC cells and the role of Hpa under conditions of hypoxia and stress (hypoxia and nutritional deprivation). (A) Exploring the anti-stress potential of TNBC cells and the role of Hpa in a hypoxic environment by proliferation analysis. (B) Exploring the anti-stress potential of TNBC cells and the role of Hpa in a hypoxic environment by colony formation assay. (C) Exploring the role of the supernatants (coculture of MDA-MB-231-Hpa-GFP cells and platelets for $30 \mathrm{~min} /$ coculture of MDA-MB-231-mock-GFP cells and platelets for $30 \mathrm{~min}$ ) under stress conditions (hypoxia and nutritional deprivation) by capillary tube formation assay. (D) Exploring the role of Hpa under stress conditions (hypoxia and nutritional deprivation). Hpa, MDA-MB-231-Hpa-GFP; mock, MDA-MB-231-mock-GFP; Hpa + PLT, MDA-MB-231-Hpa-GFP and platelets coculture for 30 min; mock + PLT, MDA-MB-231-mock-GFP and platelets coculture for $30 \mathrm{~min}$. Hpa, heparanase; TNBC, triple-negative breast cancer; PLT, platelets; HUVEC, human umbilical vascular endothelial cell. ${ }^{*} \mathrm{P}<0.05,{ }^{* *} \mathrm{P}<0.005$ and ${ }^{* * *} \mathrm{P}<0.0005$. 


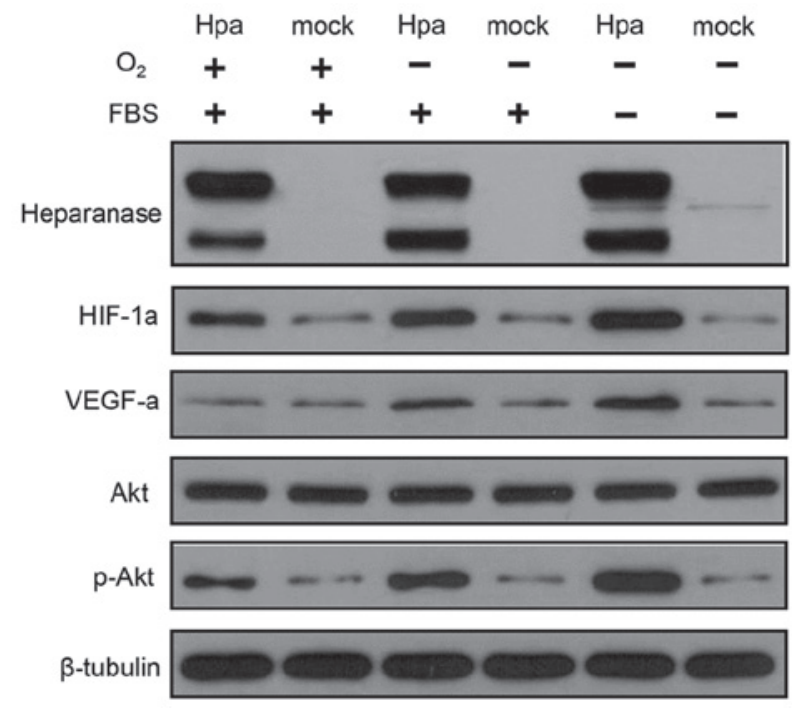

Figure 7. Gene expression and related pathways were investigated under normal, hypoxic $\left(1 \% \mathrm{O}_{2}, 5 \% \mathrm{CO}_{2}, 10 \% \mathrm{FBS}, 37^{\circ} \mathrm{C}\right)$ and stress $\left(1 \% \mathrm{O}_{2}\right.$, $5 \% \mathrm{CO}_{2}, 0 \% \mathrm{FBS}, 37^{\circ} \mathrm{C}$ ) conditions by western blot analysis. Hpa MDA-MB-231-Hpa-GFP cells; mock, MDA-MB-231-mock-GFP cells. Hpa, heparanase; HIF, hypoxia-inducible factor.

angiogenesis and metastasis (19-29). However, the role of Hpa in TNBC progression has yet to be fully revealed. In the present study, the bioinformatics analysis of TNBC cases from TCGA database indicated that: i) The expression of Hpa was significantly higher in the metastatic group compared with that in the non-metastatic group $(\mathrm{P}=0.0326)$ and high expression of Hpa was significantly associated with poor DFS $(\mathrm{P}<0.05)$ and OS ( $\mathrm{P}<0.05)$; and ii) high expression of Hpa was significantly enriched in 'BREAST CANCER METASTASIS UP', 'VEGF' and 'VEGFR1/2' pathways. Taking into consideration the previous research results from other institutes which were mentioned in the introduction, it was inferred that the intimate crosstalk between TNBC cells and PLT may be enhanced by Hpa through 'VEGF'-related pathways to promote growth, angiogenesis and metastasis in TNBC (Fig. 8) and that, to some extent, this effect of Hpa may be further enhanced by hypoxia. Further in vivo and in vitro studies were carried out to validate the hypothesis.

In the present study, the breast/lung tumor weights were significantly greater in the B/L-Hpa compared with those in the B/L-mock groups, consistently with the results of previous studies on the contribution of Hpa to tumor growth and metastasis in several types of cancer (19-29). Various tumor cells (e.g., colorectal cancer, ovarian cancer, lung cancer and melanoma, among others) have been reported to have the ability to activate PLT and promote tumor growth and metastasis $(8,13)$. In the present study, the level of PLT activation (plasma CD62P level) in tumor-bearing mice (breast tumor/lung metastasis model) was significantly elevated compared with that in the normal group, and this elevation was further enhanced by Hpa (breast tumor model), thereby indicating that TNBC cells have the ability to activate PLT, and Hpa may act as an enhancer of that ability. However, as there was no significant difference in the plasma CD62P level between L-Hpa and L-mock, it was hypothesized that this may be due to the fact that the lung metastasis model was established by tail vein injection, a method that does not absolutely mimic the physiological process of breast cancer metastasis to the lung; on the other hand, tumor cells injected by the tail vein may have a mechanical impact of the mouse circulatory system, which may be a potential interfering factor for the CD62P test results. A model efficiently mimicking the physiological process of breast cancer metastasis to the lung should be adopted to validate the CD62P results in a future study. In order to further explore the crosstalk between TNBC cells and PLT and confirm the enhancing effect of Hpa, TNBC cells (MDA-MB-231-Hpa-GFP and MDA-MB-231-mock-GFP) and human PLT were co-incubated $(30 \mathrm{~min} / 24 \mathrm{~h})$ and the coculture supernatants were collected. The results regarding the physical characteristics of the coculture supernatants (TNBC cells and PLT) indicated the following: There was crosstalk between TNBC cells and PLT, and this crosstalk significantly promoted the release of bioactive substances. As the coculture time increased, the crosstalk was further enhanced, resulting in further increase in the release of substances. These effects were enhanced by Hpa. In addition, functional tests further validated that the bioactive substances contained in the coculture supernatants significantly promoted the proliferation and migration of TNBC cells, and these effects were also enhanced by Hpa. Furthermore, the results of ELISA (in vivo) and Bio-plex analysis (in vitro) confirmed that the key growth factor was VEGF-a, further validating the results of bioinformatics analysis and our hypothesis, that the crosstalk between TNBC cells and PLT was mediated through VEGF-a to promote growth and metastasis in TNBC, which was further enhanced by Hpa.

The observation of the breast tumors indicated the following: The scabs in the B-mock group were more prominent compared with those in the B-Hpa group, which prompted us to hypothesize that the difference in the scab size may be due to the difference in the blood supply within the tumors. The observation of the lung metastases further indicated the following: The lung metastases in the L-Hpa group exhibited high number, large volume and fusion growth, which may be attributed to adequate blood supply. The lung metastases in the L-mock group were of low number and small volume, without fusion growth. However, dark red areas were identified in the L-mock group when the volume of the metastasis was large, which may be attributed to inadequate blood supply. In order to further explore whether there was a difference in the blood supply between the B/L-Hpa and B/L-mock groups, 3 related experiments were carried out. First, based on a large number of anatomical observations, the blood supply of the breast tumors relies on two major vessels (Fig. 3B); therefore, combined analysis of the abundance of the blood flow signals inside the breast tumor and the strength of the blood flow signals of the major vessels were investigated to assess the blood supply of the breast tumors. The ultrasonic examination indicated abundance of the blood flow signals inside the breast tumor, whereas the strength of the blood flow signals in the major vessels of the breast tumors were significantly higher in the B-Hpa group compared with those in the B-mock group. These findings confirmed that the blood supply was significantly higher in the B-Hpa compared with the B-mock group. Second, as the main function of blood supply is to transport oxygen to tumor tissue and, based on the confirmation that the blood supply 


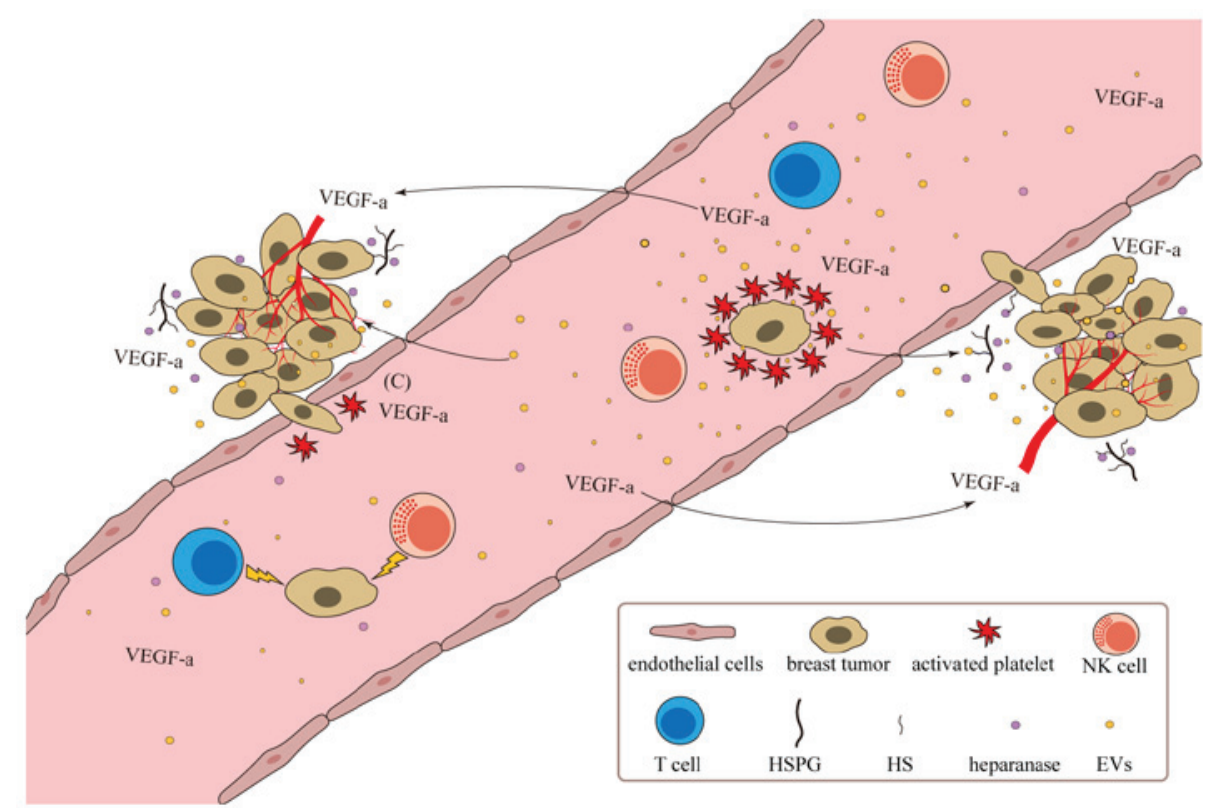

Figure 8. Hpa is the enhancer of the metastasis from TNBC cells and platelets. Hpa, on the one hand, enhancing the crosstalk between TNBC cells and platelets to increase the supply. On the other hand, conferring good tolerance to TNBC. That two aspects were both important to overcome the hypoxia even stress environment to promote the malignant progression including growth, angiogenesis, and metastasis in TNBC. Hpa, heparanase; TNBC, triple-negative breast cancer; VEGF, vascular epithelial growth factor; HS, heparan sulfate; HSPG, heparan sulfate proteoglycan; NK, natural killer; EVs, extracellular vesicles.

was significantly higher in the B-Hpa compared with that the B-mock group by ultrasonic examination, IHC (HP8) was used to explore the oxygen supply within the tumors, which revealed a significant difference in oxygen supply (hypoxia) between the B-Hpa and B-mock groups. Third, based on the confirmation of the differences in the blood and oxygen supply between the B-Hpa and B-mock groups, IHC (PAS and CD31) was performed to investigate whether angiogenesis and VM were possible explanations for these differences, and indicated that, in both breast tumors and lung metastases, angiogenesis $\left(\mathrm{CD} 31^{+}\right)$and $\mathrm{VM}\left(\mathrm{PAS}^{+}, \mathrm{CD} 31^{-}\right)$were more prominent in the B/L-Hpa compared with the B/L-mock group. Collectively, the results of these 3 related experiments indicated that Hpa increased the blood and oxygen supply of both the breast tumors and lung metastases through angiogenesis and VM, thereby contributing to tumor growth and metastasis.

Hypoxia is prevalent in solid tumors, and may be caused by insufficient vascularization, characteristic structural defects (lack of smooth muscle, incomplete endothelial lining and basement membrane), highly irregular architecture (blind ends, arteriovenous shunts and high-angle branching patterns) and functional abnormalities (42). On the other hand, it may also be caused by the high level of oxygen consumption induced by intense cell proliferation $(43,44)$ that creates an imbalance between oxygen supply and demand in solid tumors. Different tumors respond differently to hypoxia, with some tumors exhibiting inhibition of cell division and even death (45-48), while others may adapt by developing a broad range of mechanisms to accelerate malignant progression $(28,49-56)$.

In order to further explore the biological role of hypoxia in the progression of TNBC and the effect of hypoxia on Hpa, a hypoxic environment $\left(1 \% \mathrm{O}_{2}\right)$ was established in vitro. The proliferation ability of TNBC cells (Hpa/mock) and HUVECs was somewhat enhanced in hypoxia, but without a statistically significant difference. The ability of colony formation is recognized as a characteristic of tumor aggressiveness and it indicates strong adaptability and independent viability, which are crucial for tumor progression, particularly in terms of growth in a hypoxic environment or formation of metastases in a new environment. In the present study, the colony formation assay demonstrated that both hypoxia (Hpa-hypoxia $>$ Hpa-normal, mock-hypoxia >mock-normal) and Hpa (Hpa-hypoxia >mock-hypoxia, Hpa-normal >mock-normal) significantly promoted the colony-forming ability of TNBC cells, indicating that both hypoxia and the Hpa conferred good tolerance (higher tolerance to stress and increased aggressiveness) to TNBC cells. In addition, in a hypoxic environment, the ability of heparanase to promote colony formation was further enhanced in TNBC cells (Hpa-hypoxia-Hpa-normal)/Hpa-normal $>$ (mock-hypoxia-mock-normal)/mock-normal), which means that Hpa conferred good tolerance to TNBC cells, and this effect was further enhanced by hypoxia.

Oxygen, nutrient and blood supply are interlinked, so that hypoxia often coexists with nutritional deficiencies. Angiogenesis and VM are recognized as the important compensatory mechanisms to hypoxia and represent hallmarks of cancer (57). These compensatory mechanisms have been widely reported in the progression of numerous solid tumors, including TNBC (58-65). In our in vitro study, an intense stress environment was created $\left(1 \% \mathrm{O}_{2}, 5 \% \mathrm{CO}_{2}\right.$, $0 \% \mathrm{FBS}, 37^{\circ} \mathrm{C}$ ) to mimic the hypoxia and nutritional deprivation in vivo. We observed that the coculture supernatants (TNBC cells and PLT) significantly improved the reduction of vascular integrity (HUVECs), which was caused by hypoxia and nutritional deprivation, and was further enhanced by Hpa. This indicated that the crosstalk between TNBC cells and PLT contributed to angiogenesis, and this effect was enhanced by Hpa. The results of Bio-plex analysis (VEGF-a) further 
indicated that the crosstalk between TNBC cells and PLT acted through VEGF-a to promote angiogenesis and increase the blood supply in TNBC, which was further enhanced by Hpa. In this stress environment $\left(1 \% \mathrm{O}_{2}, 5 \% \mathrm{CO}_{2}, 0 \% \mathrm{FBS}\right.$, $37^{\circ} \mathrm{C}$ ), MDA-MB-231-Hpa-GFP cells exhibited a significantly increased microvascular density compared with the normal environment, but MDA-MB-231-mock-GFP cells exhibited a significantly decreased microvascular density compared with the normal environment. It was further conformed that Hpa increased the blood supply through promoting VM and conferred good tolerance to TNBC cells under conditions of extreme stress $\left(1 \% \mathrm{O}_{2}, 5 \% \mathrm{CO}_{2}, 0 \% \mathrm{FBS}, 37^{\circ} \mathrm{C}\right)$.

Numerous studies have reported that hypoxia triggers a coordinated transcriptional response, mediated primarily through the activation of the heterodimeric transcription factor HIF-1a (66-71), which induces the transcription of VEGF-a, a recognized target of HIF-1a (68,72), and significantly contributes to malignant progression in terms of growth, angiogenesis and metastasis (73-75). In ovarian and pancreatic cancer, hypoxia significantly promoted metastasis by enhancing Hpa activity $(39,76)$. However, in an extremely harsh environment (hypoxia and nutritional deprivation), the role of Hpa in TNBC has remained unknown. In the present study, it was observed that, under extreme stress conditions (hypoxia and nutritional deprivation), the expression of HIF-1a, Hpa and VEGF-a were significantly increased, which was accompanied by activation of p-AKT. Therefore, it was inferred that the HIF-1a/VEGF-a/p-AKT pathway may play a key role in this process.

In conclusion, increasing the blood supply to overcome the stress environment is important for tumor progression. However, the ability of cells to tolerate and overcome the stress environment is also important. In the present study, Hpa enhanced the crosstalk between TNBC cells and PLT to increase the supply, while also conferring good tolerance to TNBC cells, which are both important for overcoming hypoxia and a stress environment to promote the malignant progression (growth, angiogenesis and metastasis) of TNBC. The findings of the present study may help elucidate the mechanisms underlying TNBC growth, angiogenesis and metastasis from the perspective of the crosstalk between TNBC cells and PLT, and may improve our understanding of the important promoting effects and potential molecular mechanism of action of Hpa and hypoxia in the abovementioned malignant biological processes, thereby providing a theoretical basis for the effective targeted therapy of TNBC.

However, the present study had the following limitations: i) The lung metastasis model was established by tail vein injection, which does not absolutely mimic the physiological process of breast cancer metastasis to the lung, and it may be a potential factor interfering with the in vivo results. A model more closely mimicking the physiological process of breast cancer metastasis should be constructed in future studies. ii) A series of experiments were carefully conducted and documented, and we attempted to elucidate the possible underlying mechanisms. However, additional underlying mechanisms require further investigation in future studies. iii) One cell type (MDA-MB-231) was used to study the crosstalk between TNBC cells and PLT, and to study the association of Hpa with the malignant propensity of TNBC cells. Additional TNBC cell lines and other types of breast cancer or cells from other tumors are required to clearly establish the function of crosstalk between tumor cells and PLT, and the role of Hpa. Since the bioinformatics analysis based on TCGA database indicated that the statistical significance of the difference in Hpa between the metastasis and non-metastasis groups, and the correlation of Hpa with DFS and OS, were significantly higher compared with those in $\mathrm{HER} 2^{+}, \mathrm{ER}^{+}$and other types of breast cancer, without further experimental evidence we cannot conclude that this phenomenon is specific to TNBC.

In conclusion, bioinformatics analysis and experimental in vivo and in vitro studies validated that Hpa enhanced the crosstalk between TNBC cells and PLT to increase the blood supply, while also increasing the tolerance of TNBC cells to stress, which are both important for overcoming hypoxia and other stressors in the tumor microenvironment in order to promote the malignant progression, including growth, angiogenesis and metastasis of TNBC. In addition, the HIF-1a/VEGF-a/p-AKT axis may be the key pathway involved in mediating the effects of Hpa. Therefore, improving local hypoxia, anti-Hpa treatment and inhibition of PLT activation may improve the prognosis of TNBC.

\section{Acknowledgements}

The authors would like to thank Israel Vlodavsky (Cancer and Vascular Biology Research Center, Rappaport Faculty of Medicine, Technion), Bu-Xin Kou (Beijing Institute of Hepatology, Beijing Youan Hospital, Capital Medical University), Meng-Yin Chai (Beijing Institute of Hepatology, Beijing Youan Hospital, Capital Medical University, China) and Jian-Ping Fang (Glyconovo Technologies Co, Ltd. Shanghai, China) for technical support, and Wen-Bin Liu (Beijing Hospital of Traditional Chinese Medicine, Capital Medical University, China) for mechanism diagram beautification.

\section{Funding}

The present study was funded by the National Natural Science Foundation of China (grant nos. 81774039, 81873111 and 81673924).

\section{Availability of data and materials}

All the datasets generated or analyzed during the present study are included in this published article.

\section{Authors' contributions}

Conceptualization: GLZ; methodology: WJY, GLZ, KXC, XNL and MWY; software: WJY and GLZ; validation: WJY and KXC; analysis: WJY and GLZ; investigation: WJY; resources: JPL; data curation: WJY; writing-original draft preparation: WJY; writing-review and editing: GLZ, GWY and XMW; visualization: XNL; supervision: GLZ; project administration: GLZ, GWY and XMW; funding acquisition: GWY. All the authors have read and approved the final manuscript.

\section{Ethics approval and consent to participate}

All applicable international, national and/or institutional guidelines for the care and use of animals were followed. All 
procedures performed in studies involving animals were in accordance with the ethical standards and approved by the China Laboratory Animal Welfare and Animal Experimental Ethics Committee (2018030209).

\section{Patient consent for publication}

Not applicable.

\section{Competing interests}

The authors declare that they have no competing interests.

\section{References}

1. Elsawaf $\mathrm{Z}$ and Sinn H: Triple-negative breast cancer: Clinical and histological correlations. Breast Care 6: 273-278, 2011.

2. Bianchini G, Balko JM, Mayer IA, Sanders ME and Gianni L: Triple-negative breast cancer: Challenges and opportunities of a heterogeneous disease. Nat Rev Clin Oncol 13: 674-690, 2016.

3. Sharma D, Brummel-Ziedins KE, Bouchard BA and Holmes CE: Platelets in tumor progression: A host factor that offers multiple potential targets in the treatment of cancer. J Cell Physiol 229: $1005-1015,2014$

4. Cross MJ and Claesson-Welsh L: FGF and VEGF function in angiogenesis: Signalling pathways, biological responses and therapeutic inhibition. Trends Pharmacol Sci 22: 201-207, 2001.

5. Pinedo HM, Verheul H, D'Amato RJ and Folkman J: Involvement of platelets in tumour angiogenesis? Lancet 352: 1775-1777, 1998

6. LeCouter J, Gerber H and Ferrara N: The biology of VEGF and its receptors. Nat Med 9: 669-676, 2003.

7. Gil-Bernabé AM, Lucotti S and Muschel RJ: Coagulation and metastasis: What does the experimental literature tell us? Brit J Haematol 162: 433-441, 2013.

8. Gay LJ and Felding-Habermann B: Contribution of platelets to tumour metastasis. Nat Rev Cancer 11: 123-134, 2011.

9. Erpenbeck L and Schön MP: Deadly allies: The fatal interplay between platelets and metastasizing cancer cells. Blood 115: $3427-3436,2010$

10. Leblanc R, Lee S, David M, Bordet JC, Norman DD, Patil R, Miller D, Sahay D, Ribeiro J, Clézardin P, et al: Interaction of platelet-derived autotaxin with tumor integrin aVb3 controls metastasis of breast cancer cells to bone. Blood 124: 3141-3150, 2014.

11. Peterson JE, Zurakowski D, Italiano JE Jr, Michel LV, Fox L, Klement GL and Folkman J: Normal ranges of angiogenesis regulatory proteins in human platelets. Am J Hematol 85: 487-493, 2010.

12. Wojtukiewicz MZ, Sierko E, Klement P and Rak J: The hemostatic system and angiogenesis in malignancy. Neoplasia 3: 371-384, 2001

13. Bambace NM and Holmes CE: The platelet contribution to cancer progression. J Thromb Haemost 9: 237-249, 2011.

14. Buergy D, WenzF, Groden C and Brockmann MA: Tumor-platelet interaction in solid tumors. Int J Cancer130: 2747-2760, 2012.

15. Li J, Meng X, Hu J, Zhang Y, Dang Y, Wei L and Shi M: Heparanase promotes radiation resistance of cervical cancer by upregulating hypoxia inducible factor 1 . Am J Cancer Res 7 : 234-244, 2017.

16. Kelly T, Miao HQ, Yang Y, Navarro E, Kussie P, Huang Y, MacLeod V, Casciano J, Joseph L, Zhan F, et al: High heparanase activity in multiple myeloma is associated with elevated microvessel density. Cancer Res 63: 8749-8756, 2003.

17. Vlodavsky I, Friedmann Y, Elkin M, Aingorn H, Atzmon R, Ishai-Michaeli R, Bitan M, Pappo O, Peretz T and Michal I: Mammalian heparanase: Gene cloning, expression and function in tumor progression and metastasis. Nat Med 5: 793-802, 1999.

18. Vornicova O, Boyango I, Feld S, Naroditsky I, Kazarin O, Zohar Y, Tiram Y, Ilan N, Ben-Izhak O, Vlodavsky I and Bar-Sela G: The prognostic significance of heparanase expression in metastatic melanoma. Oncotarget 7: 74678-74685, 2016.

19. Zhang L, Sullivan P, Suyama J and Marchetti D: Epidermal growth factor-induced heparanase nucleolar localization augments DNA topoisomerase I activity in brain metastatic breast cancer. Mol Cancer Res 8: 278-290, 2010.
20. Gomes AM, Stelling MP and Pavão MSG: Heparan sulfate and heparanase as modulators of breast cancer progression. Biomed Res Int 2013: 1-11, 2013.

21. Sanderson RD, Yang Y, Suva LJ and Kelly T: Heparan sulfate proteoglycans and heparanase-partners in osteolytic tumor growth and metastasis. Matrix Biol 23: 341-352, 2004.

22. Edovitsky E, Elkin M, Zcharia E, Peretz T and Vlodavsky I: Heparanase gene silencing, tumor invasiveness, angiogenesis, and metastasis. J Natl Cancer Inst 96: 1219-1230, 2004.

23. Yang Y, MacLeod V, Bendre M, Huang Y, Theus AM, Miao HQ, Kussie P, Yaccoby S, Epstein J, Suva LJ, et al: Heparanase promotes the spontaneous metastasis of myeloma cells to bone. Blood 105: 1303-1309, 2005.

24. Parish CR, Freeman C and Hulett MD: Heparanase: A key enzyme involved in cell invasion. Biochim Biophys Acta 1471: M99-M108, 2001.

25. Vreys V and David G: Mammalian heparanase: What is the message? J Cell Mol Med 11: 427-452, 2007.

26. Vlodavsky I, Gross-Cohen M, Weissmann M, Ilan N and Sanderson RD: Opposing functions of heparanase-1 and heparanase-2 in cancer progression. Trends Biochem Sci 43: 18-31, 2018.

27. Ilan N, Elkin M and Vlodavsky I: Regulation, function and clinical significance of heparanase in cancer metastasis and angiogenesis. Int J Biochem Cell Biol 38: 2018-2039, 2006.

28. Vlodavsky I, Beckhove P, Lerner I, Pisano C, Meirovitz A, Ilan N and Elkin M: Significance of heparanase in cancer and inflammation. Cancer Microenviron 5: 115-132, 2012.

29. Hammond E, Khurana A, Shridhar V and Dredge K: The role of heparanase and sulfatases in the modification of heparan sulfate proteoglycans within the tumor microenvironment and opportunities for novel cancer therapeutics. Front Oncol 4: 195, 2014.

30. Freeman C, Browne AM and Parish CR: Evidence that platelet and tumour heparanases are similar enzymes. Biochem J 342: 361-368, 1999.

31. Cui H, Tan YX, Österholm C, Zhang X, Hedin U, Vlodavsky I and Li JP: Heparanase expression upregulates platelet adhesion activity and thrombogenicity. Oncotarget 7: 39486-39496, 2016.

32. Da ML, Ledaki I, Purshouse K, Haider S, De Bastiani MA, Baban D, Morotti M, Steers G, Wigfield S, Bridges E, et al: The BET inhibitor JQ1 selectively impairs tumour response to hypoxia and downregulates CA9 and angiogenesis in triple negative breast cancer. Oncogene 36: 122-132, 2017.

33. Vaupel P: The role of hypoxia-induced factors in tumor progression. Oncologist 9 (Suppl 5): S10-S17, 2004.

34. Chen X, Iliopoulos D, Zhang Q, Tang Q, Greenblatt MB, Hatziapostolou M, Lim E, Tam WL, Ni M, Chen Y, et al: XBP1 promotes triple-negative breast cancer by controlling the HIF1 $\alpha$ pathway. Nature 508: 103-107, 2014.

35. Harris AL: Hypoxia-a key regulatory factor in tumour growth. Nat Rev Cancer 2: 38-47, 2002.

36. Yamamoto Y, Ibusuki M, Okumura Y, Kawasoe T, Kai K, Iyama $\mathrm{K}$ and Iwase $\mathrm{H}$ : Hypoxia-inducible factor 1alpha is closely linked to an aggressive phenotype in breast cancer. Breast Cancer Res Treat 110: 465-475, 2008.

37. Schindl M, Schoppmann SF, Samonigg H, Hausmaninger H, Kwasny W, Gnant M, Jakesz R, Kubista E, Birner P and Oberhuber G; Austrian Breast and Colorectal Cancer Study Group: Overexpression of hypoxia-inducible factor 1alpha is associated with an unfavorable prognosis in lymph node-positive breast cancer. Clin Cancer Res 8: 1831-1837, 2002.

38. Rankin EB and Giaccia AJ: The role of hypoxia-inducible factors in tumorigenesis. Cell Death Differ 15: 678-685, 2008.

39. He X, Brenchley PEC, Jayson GC, Hampson L, Davies J and Hampson IN: Hypoxia increases heparanase-dependent tumor cell invasion, which can be inhibited by antiheparanase antibodies. Cancer Res 64: 3928-3933, 2004.

40. Saha SK, Lee SB, Won J, Choi HY, Kim K, Yang GM, Dayem AA and Cho SG: Correlation between oxidative stress, nutrition, and cancer initiation. Int J Mol Sci 18: 1544, 2017.

41. Leontieva OV and Blagosklonny MV: Yeast-like chronological senescence in mammalian cells: Phenomenon, mechanism and pharmacological suppression. Aging (Albany NY) 3: 1078-1091, 2011.

42. Dewhirst MW: Concepts of oxygen transport at the microcirculatory level. Semin Radiat Oncol 8: 143-150, 1998.

43. Vaupel P and Mayer A: Hypoxia in cancer: Significance and impact on clinical outcome. Cancer Metast Rev 26: 225-239, 2007. 
44. Semenza GL: Hypoxia-inducible factors in physiology and medicine. Cell: 399-408, 2012.

45. Yao KS, Clayton M and O'Dwyer PJ: Apoptosis in human adenocarcinoma HT29 cells induced by exposure to hypoxia. J Natl Cancer Inst 87: 117-122, 1995.

46. Graeber T, Peterson J, Tsai M, Monica K, Fornace AJ Jr and Giaccia AJ: Hypoxia induces accumulation of p53 protein, but activation of a G1-Phase Checkpoint by Low-oxygen conditions is independent of p53 Statust. Mol Cell Biol 14: 6264-6277, 1994.

47. Shimizu S, Eguchi Y, Kosaka H, Kamiike W, Matsuda H and Tsujimoto Y: Prevention of hypoxia-induced cell death by Bcl-2 and Bcl-xL. Nature 374: 811-813, 1995.

48. Schmaltz C, Hardenbergh PH, Wells A and Fisher DE: Regulation of proliferation-survival decisions during tumor cell hypoxia. Mol Cell Biol 18: 2845-2854, 1998.

49. Coquelle A, Toledo F, Stern S, Bieth A and Debatisse M: A new role for hypoxia in tumor Progression: Induction of fragile site triggering genomic Rearrangements and formation of complex DMs and HSRs. Mol Cell 2: 259-265, 1998.

50. De Bock K, Mazzone M and Carmeliet P: Antiangiogenic therapy, hypoxia, and metastasis: Risky liaisons, or not? Nat Rev Clin Oncol 8: 393-404, 2011.

51. Thiery JP, Acloque H, Huang RYJ and Nieto MA: Epithelialmesenchymal transitions in development and disease. Cell 139: 871-890, 2009

52. Vaupel P and Multhoff G: Fatal alliance of hypoxia-/HIF-1 $\alpha$ driven microenvironmental traits promoting cancer progression. Adv Exp Med Biol 1232: 169-176, 2020.

53. Sullivan R and Graham CH: Hypoxia prevents etoposide-induced DNA damage in cancer cells through a mechanism involving hypoxia-inducible factor 1 . Mol Cancer Ther 8: 1702-1713, 2009.

54. Helczynska K, Kronblad A, Jögi A, Nilsson E, Beckman S, Landberg G and Påhlman S: Hypoxia promotes a dedifferentiated phenotype in ductal breast carcinoma in situ. Cancer Res 63 . 1441-1444, 2003.

55. Graeber TG, Osmanian C, Jacks T, Housman DE, Koch CJ, Lowe SW and Giaccia AJ: Hypoxia-mediated selection of cells with diminished apoptotic potential in solid tumours. Nature 379 88-91, 1996.

56. Rokavec M, Öner MG and Hermeking H: Lnflammation-induced epigenetic switches in cancer. Cell Mol Life Sci 73: 23-39, 2016.

57. Knelson EH, Nee JC and Blobe GC: Heparan sulfate signaling in cancer. Trends Biochem Sci 39: 277-288, 2014.

58. Yue W and Chen Z: Does vasculogenic mimicry exist in astrocytoma? J Histochem Cytochem 53: 997-1002, 2016.

59. Clemente M, Pérez-Alenza MD, Illera JC and Peña L: Histological, immunohistological, and ultrastructural description of vasculogenic mimicry in canine mammary cancer. Vet Pathol 47: 265-274, 2010.

60. Sun B, Zhang D, Zhang S, Zhang W, Guo H and Zhao X: Hypoxia influences vasculogenic mimicry channel formation and tumor invasion-related protein expression in melanoma. Cancer Lett 249: 188-197, 2007.

61. Sood AK, Seftor EA, Fletcher MS, Gardner LM, Heidger PM, Buller RE, Seftor RE and Hendrix MJ: Molecular determinants of ovarian cancer plasticity. Am J Pathol 158: 1279-1288, 2001.
62. Kirschmann DA, Seftor EA, Hardy KM, Seftor REB and Hendrix MJC: Molecular pathways: Vasculogenic mimicry in tumor cells: Diagnostic and therapeutic implications. Clin Cancer Res 18: 2726-2732, 2012.

63. Seftor RE, Hess AR, Seftor EA, Kirschmann DA, Hardy KM, Margaryan NV and Hendrix MJ: Tumor cell vasculogenic mimicry. Am J Pathol 181: 1115-1125, 2012.

64. Passalidou E, Trivella M, Singh N, Ferguson M, Hu J, Cesario A Granone P, Nicholson AG, Goldstraw P, Ratcliffe C, et al: Vascular phenotype in angiogenic and non-angiogenic lung non-small cell carcinomas. Br J Cancer 86: 244-249, 2002.

65. Folberg R, Hendrix MJ and Maniotis AJ: Vasculogenic mimicry and tumor angiogenesis. Am J Pathol 156: 361-381, 2000.

66. Semenza GL: Defining the role of hypoxia-inducible factor 1 in cancer biology and therapeutics. Oncogene 29: 625-634, 2010.

67. Semenza GL: HIF-1 mediates metabolic responses to intratumoral hypoxia and oncogenic mutations. J Clin Invest 123 3664-3671, 2013.

68. Maxwell PH, Dachs GU, Gleadle JM, Nicholls LG, Harris AL, Stratford IJ, Hankinson O, Pugh CW and Ratcliffe PJ: Hypoxia-inducible factor-1 modulates gene expression in solid tumors and influences both angiogenesis and tumor growth. Proc Natl Acad Sci USA 94: 8104-8109, 1997.

69. Wenger RH, Stiehl DP and Camenisch G: Integration of oxygen signaling at the consensus HRE. Sci Stke 2005: re12, 2005.

70. Chen Z, He X, Xia W, Huang Q, Zhang Z, Ye J, Ni C, Wu P, Wu D, $\mathrm{Xu}$ J, et al: Prognostic value and clinicopathological differences of HIFs in colorectal cancer: Evidence from meta-analysis. PLoS One 8: e80337, 2013

71. Fan L, Dong W, Jiang C, Qian Q and Yu Q: Role of Hypoxiainducible factor- $1 \alpha$ and Survivin in colorectal carcinoma progression. Int J Colorectal Dis 23: 1057-1064, 2008.

72. Semenza GL: Targeting HIF-1 for cancer therapy. Nat Rey Cancer 3: 721-732, 2003.

73. Tseng W, Yang S, Lai C and Tang C: Hypoxia induces BMP-2 expression via ILK, Akt, mTOR, and HIF-1 pathways in osteoblasts. J Cell Physiol 223: 810-818, 2010.

74. Pugh CW and Ratcliffe PJ: Regulation of angiogenesis by hypoxia: Role of the HIF system. Nat Med 9: 677-684, 2003.

75. Manalo DJ, Rowan A, Lavoie T, Natarajan L, Kelly BD, Ye SQ, Garcia JG and Semenza GL: Transcriptional regulation of vascular endothelial cell responses to hypoxia by HIF-1. Blood 105: 659-669, 2005.

76. Gao J, Su L, Qin R, Chang Q, Huang T and Feng Y: Transfection of antisense oligodeoxynucleotide inhibits heparanase gene expression and invasive ability of human pancreatic cancer cell in vitro. J Huazhong Univ Sci Technolog Med Sci 26: 72-74, 2006.

This work is licensed under a Creative Commons Attribution-NonCommercial-NoDerivatives 4.0 International (CC BY-NC-ND 4.0) License. 